\title{
Multivariate cyclical data visualization using radial visual rhythms: A case study in phenology analysis
}

\author{
Greice C. Mariano $^{\mathrm{a}, *}$, Vanessa G. Staggemeier ${ }^{\mathrm{b}}$, Leonor Patricia Cerdeira Morellato ${ }^{\mathrm{b}}$, \\ Ricardo da S. Torres ${ }^{\text {a }}$ \\ a Institute of Computing, University of Campinas (UNICAMP), Campinas, SP, Brazil \\ ${ }^{\mathrm{b}}$ UNESP - São Paulo State University, Institute of Biosciences, Department of Botany, Phenology Laboratory, Rio Claro, SP, Brazil
}

\section{A R T I C L E I N F O}

\section{Keywords:}

Phenology visualization

Multivariate time series

Radial visualization

Visual rhythm

\begin{abstract}
A B S T R A C T
Phenology is a traditional science that investigates the periodic phenomena of plants and animals and their relations to environmental conditions. Typically plant phenological studies are based on observations made by phenology experts in the field over time and the correlation with climate data collected by weather sensors. Although within the visualization community several approaches have been proposed for visualizing data that vary over time, many of them have a specific purpose and cannot be applied to phenology studies. Besides that, phenology experts increasingly need tools for managing appropriately long-term time series with many variables of different data types, as well as to identify cyclical temporal patterns. In this work, we propose a novel approach to visualize phenological data by combining radial visual structures along with visual rhythms. Radial visual structures are used to provide contextual insights regarding cyclical phenomena, while the visual rhythm encoding is used to summarize long-term time series into compact representations. We developed, evaluate, and validate our proposal with phenology experts using plant phenology direct observational data both at individuals and species levels.
\end{abstract}

\section{Introduction}

Phenology is an important science that investigates the recurrent life cycles from living beings (plants and animals) and their relations to the environment (Schwartz, 2003). Traditional phenological studies are typically based on direct observations made by experts in the field over time. For example, phenology experts usually monitor several individuals - from the same or different species - periodically, at monthly intervals (Morellato et al., 2013; Morellato et al., 2010b).

Generally, in the context of plant phenology, experts need to choose a sampling method, which defines how individuals and species will be sampled (spatial distribution) and then, to choose an observation method for tracking the temporal distribution of events or phenophases, and for scoring or quantifying the phenological activity over time ( $\mathrm{d}^{\prime} \mathrm{E}$ ça Neves and Morellato, 2004; Morellato et al., 2016; Morellato et al., 2010b). These methodological procedures define how the observations will be converted into useful data. For example, for each individual, experts have to observe and take notes about the changes on phenophases such as the appearance of new leaves, development of flowers and fruits, as well as the leafout (d'E ça Neves and Morellato, 2004; Talora and Morellato, 2000; Morellato et al., 2010b). One way to record the changes on phenophases relies on the use of categories represented by numerical values to estimate intensity of occurrence for each phenophase (d'E ça Neves and Morellato, 2004; Morellato et al., 2010b). Thus, experts use these numerical values to analyze and to identify temporal patterns of individuals at the same species, or across species in a community. Some examples include dates of flowering or fruiting peaks (Talora and Morellato, 2000) or number of flowers and fruits produced (Athayde and Morellato, 2014).

Furthermore, phenology studies are usually targeted towards the investigation of the correlation of phenological phenomena with climate data. The objective is to answer questions about environmental changes and their impact on plants (Morellato et al., 2016). In this scenario, a noticeable challenge faced by phenology experts is how to organize and to analyze diverse multidimensional cyclical temporal data (time series), considering different timescales.

A common approach used for data organization relies on the use of database-oriented solutions, such as relational databases, to store plant phenology data (Dierenbach et al., 2013; Mariano et al., 2016b). Despite their success, the need of visual analytics tools still persists. Although several approaches have been proposed to visualize time series (Aigner et al., 2011), most of them are not appropriate or applicable to

\footnotetext{
* Corresponding author.

E-mail address: gmariano@ic.unicamp.br (G.C. Mariano).
} 
phenology data, as they are not able: (i) to handle long-term series with many variables of different data types and one or more dimensions and (ii) to identify cyclical temporal patterns and the associated environmental drivers. Furthermore, visualization tools should support data analysis under different perspectives. For example, a detailed view focusing on individual and at larger scales, considering data about species and communities.

In this paper, we address these issues by introducing one suitable alternative for visualizing data related to phenology studies. Our approach is based on using a radial layout along with visual rhythms (VR). The use of a radial structure aims to provide contextual insights regarding cyclical temporal phenomena. The use of radial representation has been successfully applied on phenology studies instead of the linear representations through the circular statistics analyses (Morellato et al., 2000; Morellato et al., 2010a). The circular analyses and representation is a particular way to represent temporal data with no true zero or starting point (Fisher et al., 1993). Traditionally, VR representations were proposed to encode temporal change from video data using linear pixel sampling (Kim et al., 1998; Ngo et al., 1999). In more recent studies, VR representations have been used to visualize temporal properties from digital images (Almeida et al., 2016); Leite et al., 2016). The main advantages of this approach rely on efficiency aspects, as the compact representations generated are usually less costly in terms of storage and processing. With this in mind, the main novelty here is on exploiting the use of VR representations to encode conventional phenological numerical data. To the best of our knowledge, this is the first initiative dedicated to the use of VR representations to encode conventional numerical data.

In summary, the key contribution of this work is the specification and the implementation of a visualization approach using radial layouts, along with visual rhythm representations, to explore large volumes of multidimensional temporal data, usually associated with different types of variables. The objective is to support the detection of cyclical patterns. We demonstrate the potential use of our tool in the context of phenology studies using data from the e-phenology project as a case study (Alberton et al., 2014; Mariano et al., 2016b).

We developed ten prototypes that were separated and validated in two groups with phenology experts (potential users of our tool), such as: (i) six prototypes were created to represent phenological data about individuals - which we call data visualization at detailed level and (ii) four prototypes were created to represent phenological data about species - which we call data visualization at summarization level. We also included two prototypes based on linear bar graphs (one for each level), which represent the typical visual charts usually created by phenology experts in their temporal analysis studies. All prototypes encode, visually, data associated with research questions related to the correlation among phenology phenomena and climatic conditions. The first results obtained show that our proposal is able to support the analysis of phenological data, mainly when the experts are interested in understanding the relations among multiple variables associated with plant life cycle events. Furthermore, we were able to identify the need of creating interaction mechanisms to support phenology experts in tasks such as data filtering (i.e., by year, phenophase, and type of data) and data browsing. The objective is to improve their experience in the understanding of complex temporal change patterns.

This paper extends the work published in Mariano et al. (2016a). In that paper, we introduced the idea of combining visual rhythms and radial structures to encode phenological data. Different from that paper, we present here a comprehensive formal description of the combination procedure, concerning the use of both phenological and climate data, from a database perspective. The objective is to guide researchers and developers in the creation of novel realizations and extensions of the proposed approach for managing multidimensional data in different applications. Lastly, another novelty of this work refers to the description of user-centered evaluation procedures aiming to validate different prototypes in the context of real-world phenology studies. To the best of our knowledge, this is one of the first works in the literature to describe experiments with phenology experts aiming to validate information visualization tools in a real scenario setting.

The remainder of this paper is organized as follows. Next section presents related work. In the following, we present our case study on the visualization of data produced in the context of the e-phenology project. Next, we present an overview of our approach, followed by the discussion upon the usage scenarios and introduce our generic prototype proposal. We then present the created prototypes and discuss about user-centered evaluations performed with phenology experts. Finally, we offer our conclusions and directions for future work.

\section{Related work}

Plant phenology studies are based on the observation of individuals and/or species and the correlation of data related to their life cycle with climate variables (Morellato et al., 2016; Schwartz, 2003). Recent plant phenology studies are not only concerned with the analysis of data from direct plant observations in the field (Morellato et al., 2010b), but also consider near-remote data derived from sequential vegetation images taken by digital cameras (Alberton et al., 2014, 2017). We are not addressing remote phenology in this paper, but the proposal of appropriate visualization strategies for this kind of data in the future is of paramount importance.

There are several approaches to visualize time series as shown in the seminal book written by Aigner et al. (2011). Most of those initiatives, however, have limitations to visualize long time series of different types and domains, i.e., they are not robust enough to visualize multidimensional temporal data. In this sense, researchers have developed visualization techniques for specific purposes. In Arsenault et al. (2004), Ferreira et al. (2011), Horn et al. (2009) and Leite et al. (2016), for example, we found different biodiversity systems with some features to visualize data varying over time in their context. None of them combines radial structures with visual rhythm representations at the same time. The work of Leite et al. (2016), however, takes advantage of visual rhythms and special color mapping strategies to depict changes of image-related phenological features over time.

In the following section, we discuss related work upon the use of visual rhythm representations and radial layouts.

\subsection{Visual rhythm}

In general, a video can be physically viewed as a set of images (usually referred to as frames) sequentially ordered over time (Ngo et al., 1999). Each image that composes a video can be sampled using different criteria (e.g., based on a vertical, horizontal, or a diagonal line). Features may be extracted from those samples and later combined into an image in order to encode video changes over time. The image generated by this methodology is called Visual Rhythm (Ngo et al., 1999; Chung et al., 2000). The main objective is to facilitate video analysis by reducing the storage needs associated with features extracted from images and speeding up video processing algorithms (Guimarães et al., 2003).

Formally, a visual rhythm is the simplification of one video $V$ at domain $2 \mathrm{D}+t$ to domain $1 \mathrm{D}+t$, as presented in Fig. 1 . Each video $V$ has $T$ frames, where each frame $F$ has the same height and width, therefore $V=\left\{F_{t}\right\}, t \in[1, T]$ (Fig. 1a). Each frame $F_{t}$ is characterized by a set of pixels - samples - $(x, y)$ associated with a time instant $t$, then, $F_{t}=\{(x, y, t)\} . F_{t}$ is also known as a video cut (Chung et al., 2000) (Fig. 1b).

In order to analyze the video from the temporal point of view, new cuts can be defined in $\left\{F_{t}\right\}$ using different sampling strategies (e.g., based on vertical, horizontal, or diagonal lines). Fig. 1c illustrates the use of a horizontal sampling approach. In this example, each $F_{t}$ is transformed into a column on a new image, which represents the video visual rhythm (Fig. 1c). Therefore, the visual rhythm (VR) of a video is 
(a)

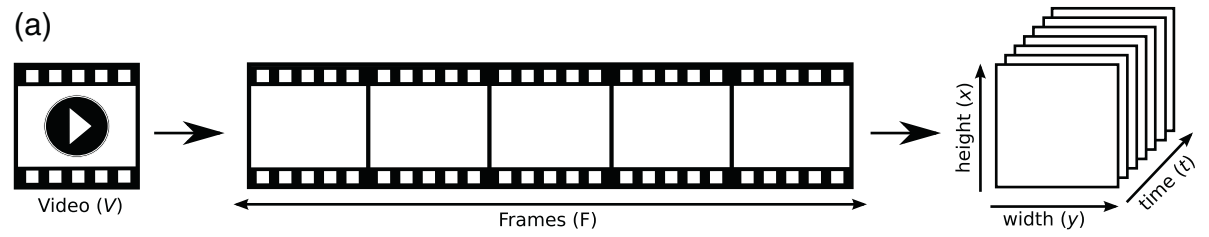

(b)

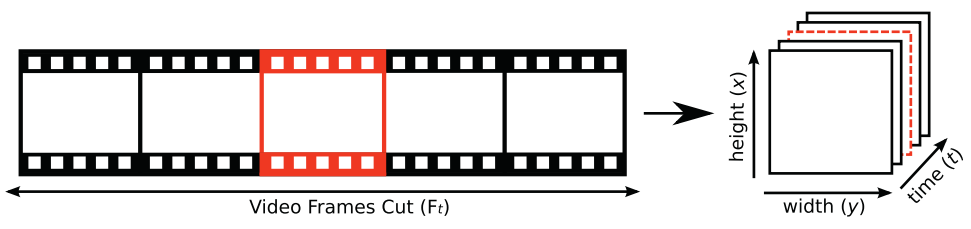

(c)

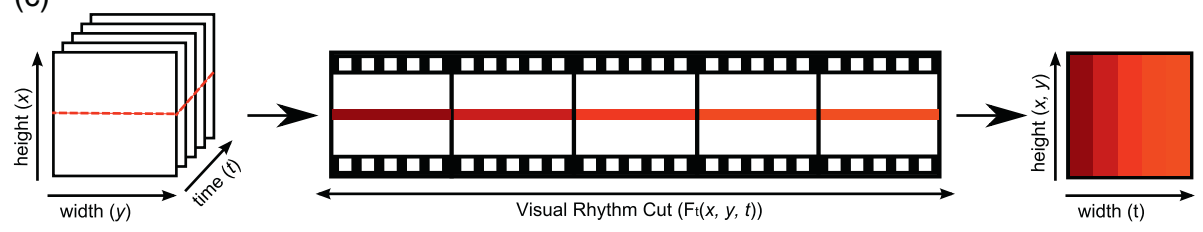

Fig. 1. Visual rhythm examples of a video, a video cut, and a visual rhythm representation.

given by $V R=\left\{F_{V R}(i, t)\right\}=\left\{F_{\text {sampling }}(x(z), y(z), t)\right\}, i \in\{0,1,2, \ldots\}$ and $t \in T$, where $F_{\text {sampling }}(x, y, t)$ is a spacial reduced version from $F_{t}(x, y, t)$, and $x(z)$ and $y(z)$ are functions of one dimension of independent variable $z$, where $z$ and $t$ refer to vertical and horizontal axis, respectively (Kim et al., 1998).

The visual rhythm approach has been used with success in different applications, such as: video cut detection, identification of transitions and flashes (Guimarães et al., 2003) content-based video retrieval (Ngo et al., 2003; Valio et al., 2011); social analysis (Hochman and Schwartz, 2012). More recently, some studies have been using visual rhythms in Biology (Matuszewski et al., 2012; Matuszewski et al., 2013), including remote phenology (Almeida et al., 2013a, 2013b, 2016) and hyperspectral remote sensing image analysis (dos Santos et al., 2014). In this paper, we construct a visual rhythm representation for time series associated with numerical data stored in relational tables derived from traditional direct phenological observation.

\subsection{Radial visualization}

Radial visualization is the term used to describe a visualization system that uses circular or elliptical layout to display data. This visualization layout has been increasingly used for many purposes (Draper et al., 2009). For temporal data, for example, the radial display has been widely used to build visualizations, such as spiral graph (Weber et al., 2001), circle view (Keim et al., 2004), circle segments (Ankerst et al., 1996), TimeRadarTrees (Burch and Diehl, 2008), and axes-based visualization (Tominski et al., 2004). In another research venue, this approach is combined with other techniques. Some examples include the use of the radial visualization for geoscience observation data (Li et al., 2015) and of the radial projection for geoinformation (Drocourt et al., 2011). Finally, phenology has used radial representations instead of the linear representations thorough the circular statistics analyses (Morellato et al., 2000; Morellato et al., 2010a). The circular representations is a common way to represent temporal data with no true zero or starting point (Fisher et al., 1993). Many other applications of radial visualization are introduced by Draper et al. (2009).

Inspired by these works, we also use the radial layout to represent data from phenology studies. Our approach encodes time dimension using concentric circles (Daassi et al., 2013) and show multivariate data using a similar concept presented in the circle view (Keim et al., 2004), and circle segments (Ankerst et al., 1996) approaches. Furthermore, we also use visual rhythm to summarize a large volume of data, building a visualization that enables the identification of cyclical patterns related to phenology data.

According to Draper et al. (2009), many types of radial visualization exist and they can be combined to produce novel visualizations, such as the case of Masoodian et al. (2013), which combines radial structure with pie chart to visualize the proportions of total energy used by one type of device across a measured period of time. Both Carlis and Konstan (1998) and Weber et al. (2001) proposed the use of spirals structures to show the seasonality of data. In our proposal, we also use the concept of radial structure to organize time-varying phenological data.

\section{Case study: the e-phenology project}

\subsection{Context}

Phenology data are periodically collected from individuals located in an observation area named site and the data values of observation vary according to the sampling method used by experts (Morellato et al., 2010b). In our research, we use data collected in the context of the long term Cerrado Phenology (Camargo et al., 2011) and the multidisciplinary e-phenology project (Mariano et al., 2016b; Alberton et al., 2014, 2017).

The numerical data considered in the context of the e-phenology project is related to a core study site - a cerrado savanna area - of about 260 ha located at Itirapina, São Paulo State, southeastern Brazil. In the cerrado core site about 2122 individuals were sampled over 36 transects, tagged, identified, and are observed monthly for phenology changes since 2004 up today (Camargo et al., 2011; Vogado et al., 2016; Alberton et al., 2014). The observers assign a score value that represents the intensity of plant phenophases (e.g., flower bud, leaf flush, and fruit). The scores range from 0 to 2 (Morellato et al., 2010b; Camargo et al., 2011; Vogado et al., 2016), where: 0 means that the phenophase was not identified; 1 means that the phenophase was present in up to $50 \%$ of the plant, or low presence, and 2 means that phenophase was present in more than $50 \%$ of the plant up to $100 \%$ or high presence. A database model was recently proposed to handle phenological data, and for more details regarding the data model of the database designed for the e-Phenology project, readers may refer to Mariano et al. (2016b). These data (phenophase, date, and values) are stored into a single table named dataPhenology associated with one 
individual, i.e., each tuple of this table refers to one phenophase observed for one individual and for each individual we have six tuples in the table. Furthermore, the database also contains tables to store weather data for different sensors (table weatherData).

\subsection{Data types}

Basically, the data managed in the context of the e-phenology project can be classified into two types: (i) non-temporal and (ii) temporal data. These data are stored into conventional databases (Mariano et al., 2016b). Non-temporal data are related to species taxonomy, location, and ecological information about species and individuals. These data are important to contextualize and geographically locate temporal data.

Temporal data, in turn, refer to:

- Phenology data: are obtained from direct on-the-ground observation of plant's life cycles. These phenological data of events such as flowering, fruiting, and leafing can be collected daily, fortnightly, or monthly (as in the case of the e-phenology project), over the years (Camargo et al., 2011; Vogado et al., 2016; Alberton et al., 2014). The observer can score the phenophase as: 0 (absence), 1 (between 1 and $50 \%$ or low activity) and 2 (above $50 \%$ up to $100 \%$ or peak of activity). Fig. 2 shows two examples of numerical time series: (a) univariate time series and (b) multivariate time series. In these examples, the data illustrated refer to phenology observations of one individual of Myrcia guianensis species collected monthly during 2012 in the Cerrado core study site at Itirapina, São Paulo, Brazil
(Vogado et al., 2016). The example in Fig. 2a shows a time series with only one variable of the set $V$. This variable is the flower bud phenophase, whose domain varying over time $T$ are integer numbers $(0,1$, or 2$)$. In turn, Fig. $2 \mathrm{~b}$ shows a time series with three variables for the set $V$ : flower bud, anthesis, and leaf flush. In this example, for each variable from $V$, the values have the same domain from previous example and are associated with the same time instant $t_{i}$ in $T$.

- Climate data: are obtained by weather sensors installed in the site where the phenology study is performed. The main objective with these data is to monitor the local information about amount of rainfall, wind speed, solar radiation, and relative humidity. These data are usually collected daily in predefined time intervals. In the case of e-phenology project, the sensors collect data every $5 \mathrm{~min}$, during $24 \mathrm{~h}$ a day, every day of year.

\subsection{Target analysis problem}

Usually, in plant phenology studies experts want to identify cyclical temporal patterns both, at individual level, as well as at species and community levels. A critical problem faced relies on the lack of appropriate tools to support these analyses. Although there are many visualization techniques for exploring time series, there is no standard tool with which phenology experts may explore their data. Furthermore, comparative studies involving multiple variables and dimensions are even more difficult to be performed (Morellato et al., 2010a).

For instance, to investigate the climate drivers for plant phenology (a)

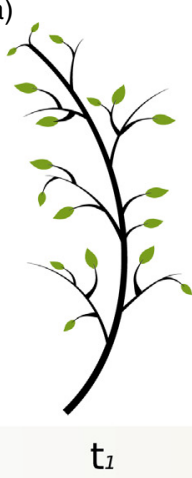

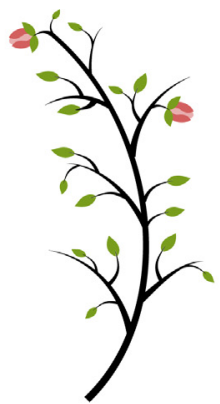

$\mathrm{t}_{2}$

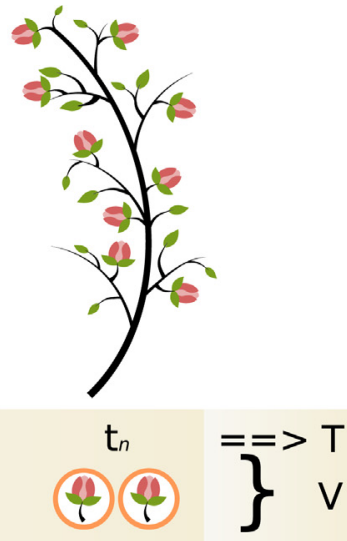

(b)

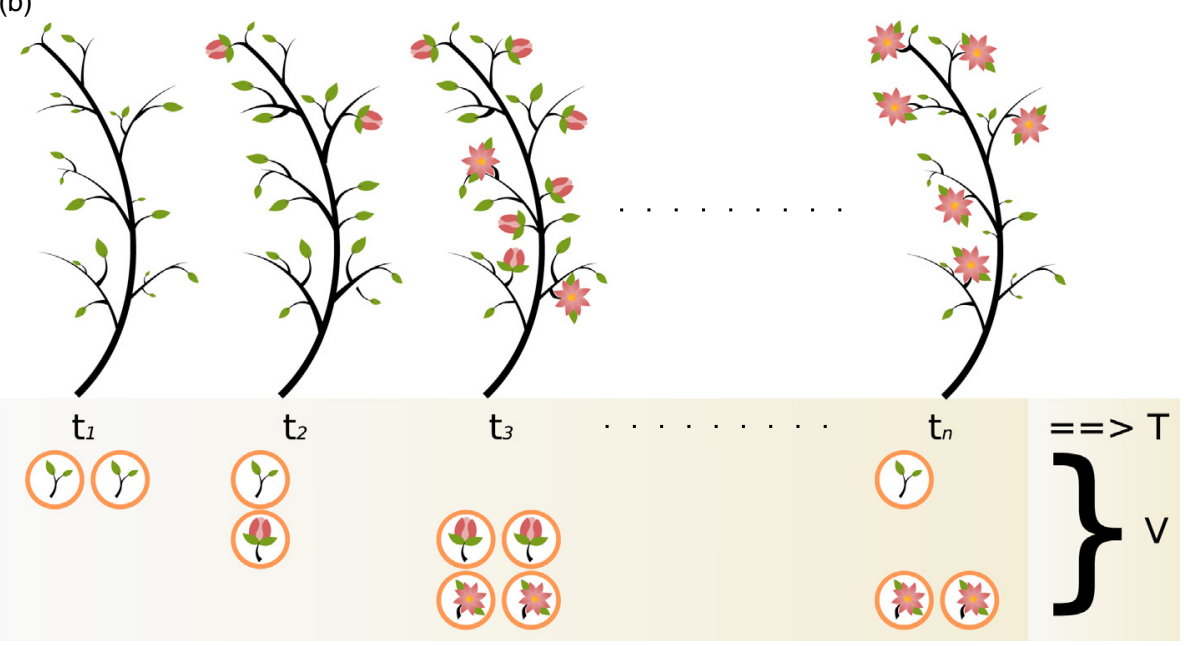

Fig. 2. Example of (a) univariate and (b) multivariate time series. 
changes, experts have to produce a graphic joint visualization that integrates both, phenology and climate data. Some examples of typical graphics used in phenology studies can be found in Staggemeier and Morellato (2011), Camargo et al. (2011), and Alberton et al. (2014).

In summary, phenology experts spend much time to organize useful data and to create appropriate graphical representations that might be used to identify existing temporal relations. According to plant phenology experts, generally, the main problems related to the most commonly used data visualization approaches are:

1. There is no standard approach or tool with which they can visualize and analyze temporal data.

2. The graphics used are static and commonly do not support multiple variables and/or multidimensional analysis.

3. The graphics used are created by using more than one software in several cases, and frequently have to be combined manually using graph editors to reach the visualization goal. This is a costly and time-consuming task as it demands a lot of efforts and training in many softwares at the same time.

\section{Proposed approach}

We present a novel approach for visualizing phenological data using a radial visual structure along with visual rhythms. Our objective is to support the knowledge discovery process by allowing the analysis of multidimensional cyclical data.

\subsection{Overview}

The main objective of this work is to propose a visualization approach with the objective of supporting the identification of cyclical temporal patterns from long time series. More specifically, we want to investigate, for the first time, the use of visual rhythms in the analysis of conventional numerical, on-the-ground direct observational data used in phenology studies. In this context, Fig. 3 presents an overview upon our proposal.

In Fig. 3 component $A$, we have a representation of temporal data used in phenology studies and explored in our present proposal. We consider the analysis of cyclical data associated with numerical (data related to both direct on-the-ground observations and climatic information). Once the data are obtained, they are processed and stored into conventional database (Fig. 3 component $B$ ). Next, visual rhythmbased representations may be extracted from both numerical and climate data. This step is illustrated by component $C$ in the Fig. 3. The visual rhythm representation is based on a two-dimensional image, which is expected to summarize the most important properties of the multidimensional data. Since our interest is to support the identification of cyclical temporal patterns, we use a radial visualization structure (Fig. 3 component $D$ ). Finally, as shown in Fig. 3 component $E$, we encode the visual rhythm representation into a radial structure.

\subsection{Visual rhythms for phenological data}

In our proposal, we want to explore the use of visual rhythms for conventional numerical data. In the following, we detail how to represent these data using visual rhythms.

Phenology studies typically are based on the analysis of large volumes of temporal and cyclical data. For example, those studies often consider the observation of the life cycles of thousands of plants over time (Dierenbach et al., 2013). Common research questions addressed in those studies refer to the analysis of numerical data related, for example, to when (time of the year), for how long (duration), and at which intensity a given event (e.g., leafing or flowering) has occurred at a specific region (Talora and Morellato, 2000; Morellato et al., 2010b). The most typical approach to store conventional numerical data associated with phenology studies relies on the use of relational databases
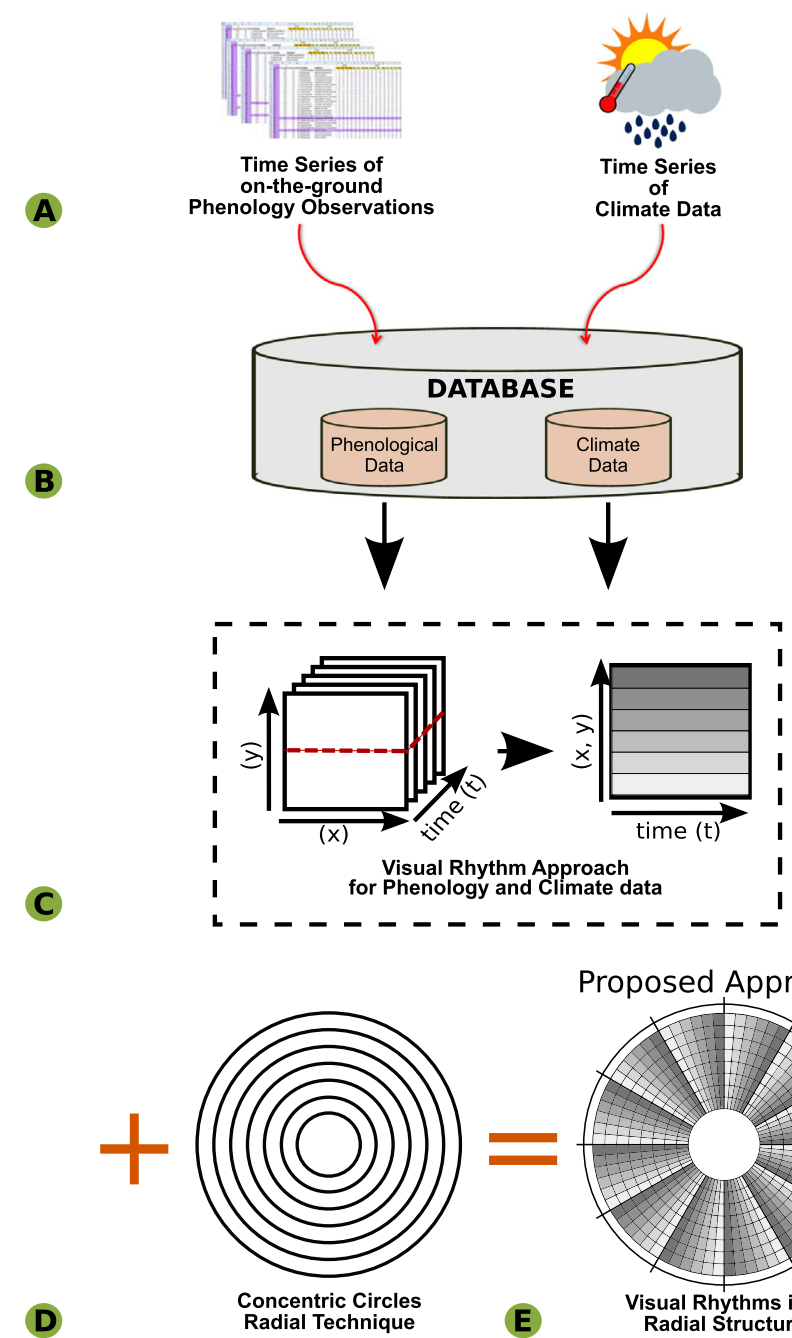

Proposed Approach

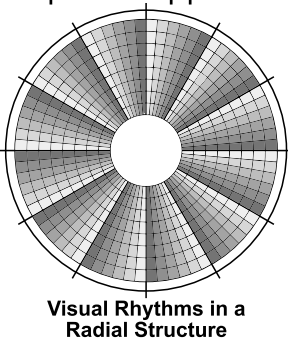

Fig. 3. Overview of the visualization technique proposed for textual and climate data.

(Mariano et al., 2016b; Dierenbach et al., 2013).

In this work, we propose the use of visual rhythm representations to encode numerical temporal data stored in relational tables. The objective is to support the identification of cyclical patterns in numerical data related to phenological studies. In order to represent temporal changes of numerical data, we use the same idea of original visual rhythm approach wherein a video is decomposed into multiple images. Without loss of generality, we assume that it is possible to encode phenological temporal data into a set of tables ordered in time.

Let $R\left(A_{0}, A_{1}, \ldots, A_{m}, T\right)$ be a relation (table), where $A_{i}(0 \leq i \leq m)$ is a numeric attribute and $T \in \mathbb{N}$ is an attribute associated with time instants. Without loss of generality, the relation $R$ can be decomposed into several smaller tables by performing selections based on the values of the time attribute. For example, Fig. 4 illustrates the decomposition of relation $R$ based on the selection of tuples (rows of the table) associated with different timestamps $t(1 \leq t \leq n)$. In the example, relations $R_{t}$ refer to the set of tuples whose the value of attribute $T$ is equal to $t$. Using the relational algebra notation, $R_{t}=\sigma_{T=t}{ }^{R}$, where $\sigma$ is the selection operator. ${ }^{1}$

Each $R_{t}$ has its width equal to the number of numerical attributes $(m)$ and height equal to the number of tuples associated with the same time instant $t$. In phenology studies, for example, $A_{i}$ may refer to the

\footnotetext{
${ }^{1}$ For more details upon the typical relational algebra operators, readers may refer to Elmasri and Navathe (2010).
} 

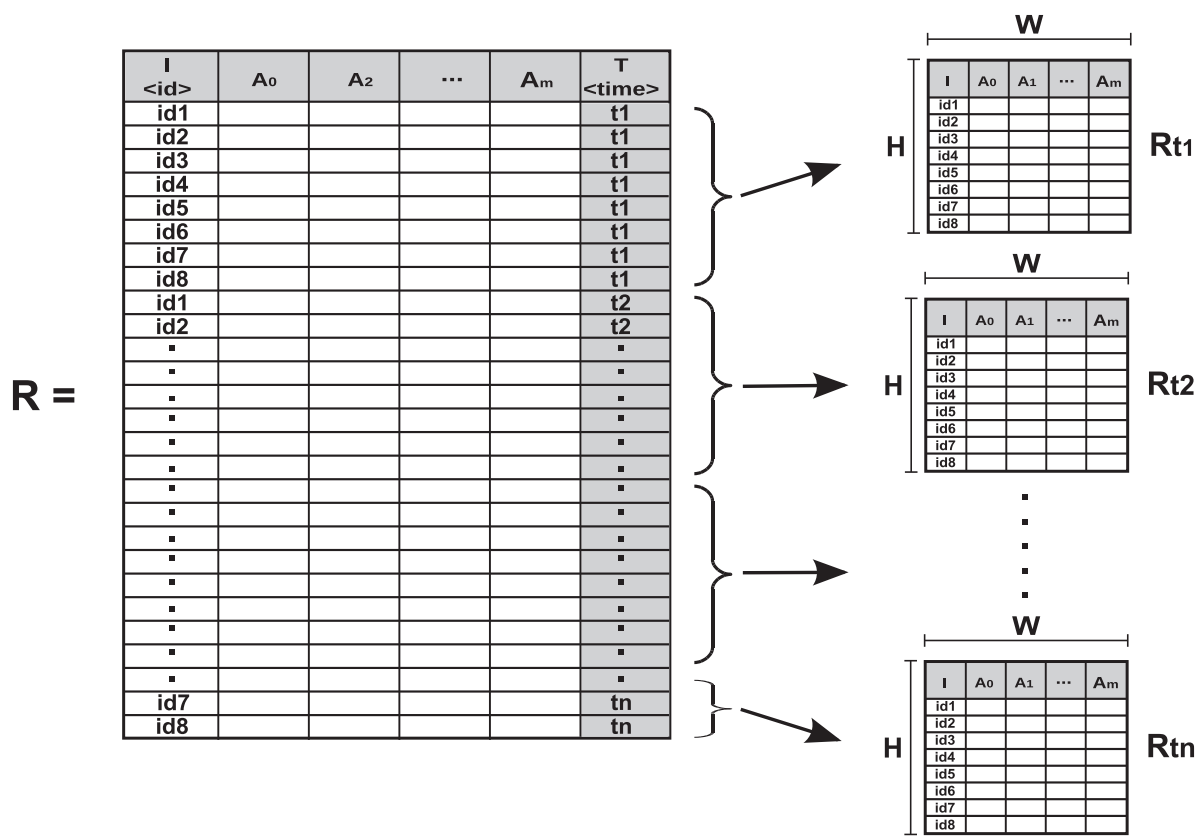

Fig. 4. Decomposition of relation $R$ into several relations $R_{t}$, where $t$ refers to a timestamp.

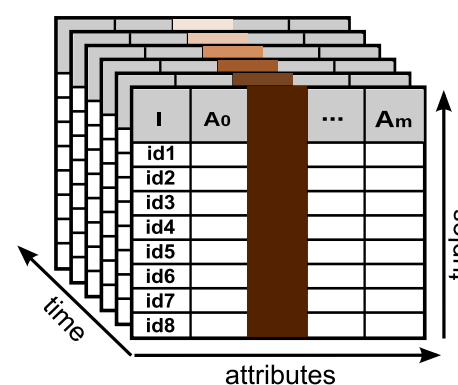

(a)

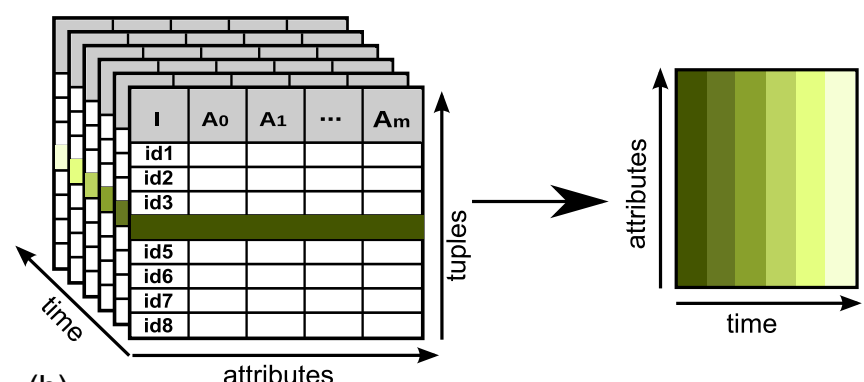

(b)

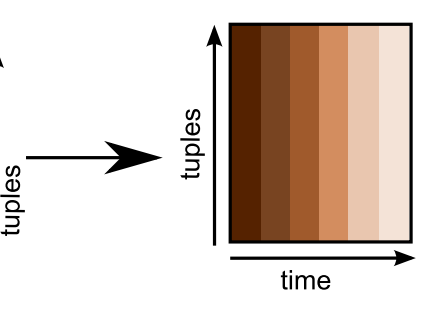

(a)

Fig. 5. Examples of visual rhythm creation based on: (a) projections (vertical cuts) and (b) selections (horizontal cuts)

phenophase intensity (e.g., for flower bud or anthesis) observed for different individuals over time. A specific $R_{t}$, in turn, may refer to the set of the phenophase intensities observed for all individuals at timestamp $t$.

The decomposition of relation based on time information leads to a set of matrices that can be used to generate visual rhythm representations. For example, Fig. 5a shows the creation of a visual rhythm representation based on column information. Following our previous example, supposing we are interested in studying changes of a particular phenophase (defined by attribute $A_{i}$ ) over time. In this case, $R_{v}(t, z)=\left\{\pi_{A_{i}}\left(R_{t}\right)\right\}, t \in[1, n]$ and $z \in[1, H]$, where $n$ and $H$ are its width and height, respectively, and $\pi$ stands for the relational algebra

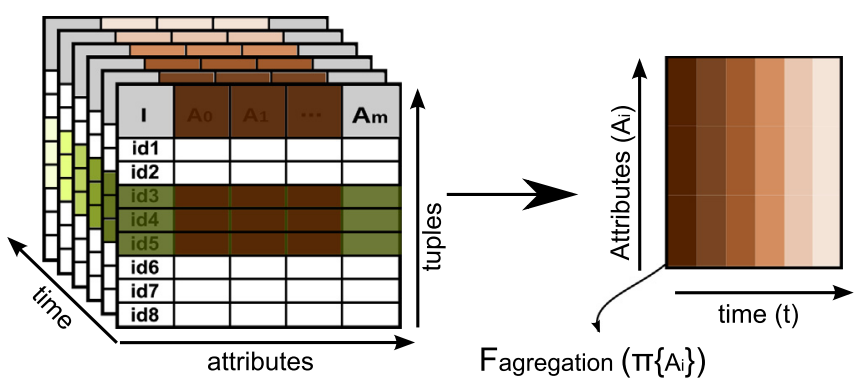

Fig. 6. Example of the use of the visual rhythm approach in tabular data using aggregation operations.

projection operator that returns a subset of $R_{t}$, restricted to the set of attributes defined by $A_{i}$.

Suppose now that we are interested in observing changes on intensities of all phenophases for a particular individual $\langle i d\rangle$. In order to support this analysis, we can create a visual rhythm representation based on multiple horizontal selections (one for each relation $R_{t}$ ). Formally, $R_{v}(t, z)=\left\{\pi_{\mathrm{A}}\left(\sigma_{I=<i d}>\left(R_{t}\right)\right)\right\}, t \in[1, n]$ and $z \in[1, W]$, where $\mathbb{A}$ is a set of attributes associated with different phenophases. In this case, the numerical and time attributes will form $y$-axis and $x$-axis, respectively, into visual rhythm image as shown Fig. 5b.

Another way explored in this paper to build visual rhythms from relational tables relies on the selection of more than one value for each attribute and more than one attribute. Thus, in addition to projection and selection operations we also have to use an aggregation function to summarize data, such that $\left.\left.R_{v}(t, z)={ }_{\left\{A_{i}\right.}\right\} F_{\text {agregation }}\left(\pi_{\left\{A_{i}\right.}\right\}\left(\sigma_{\text {condition }}\left(R_{t}\right)\right)\right), t \in$ $[1, W]$ and $z \in[1, H]$, where $z$ on this case is a multidimensional variable. Typical aggregation functions used include count and average. Fig. 6 illustrates this example.

\section{Implementation details}

Fig. 7 shows the basic idea behind our visualization approach for phenology data. The main objective is to develop a tool that supports the joint visualization of all variables typically involved in phenology studies. In Fig. 7, we represent some of them. We are using the idea of concentric circles divided into segments that are associated with the 


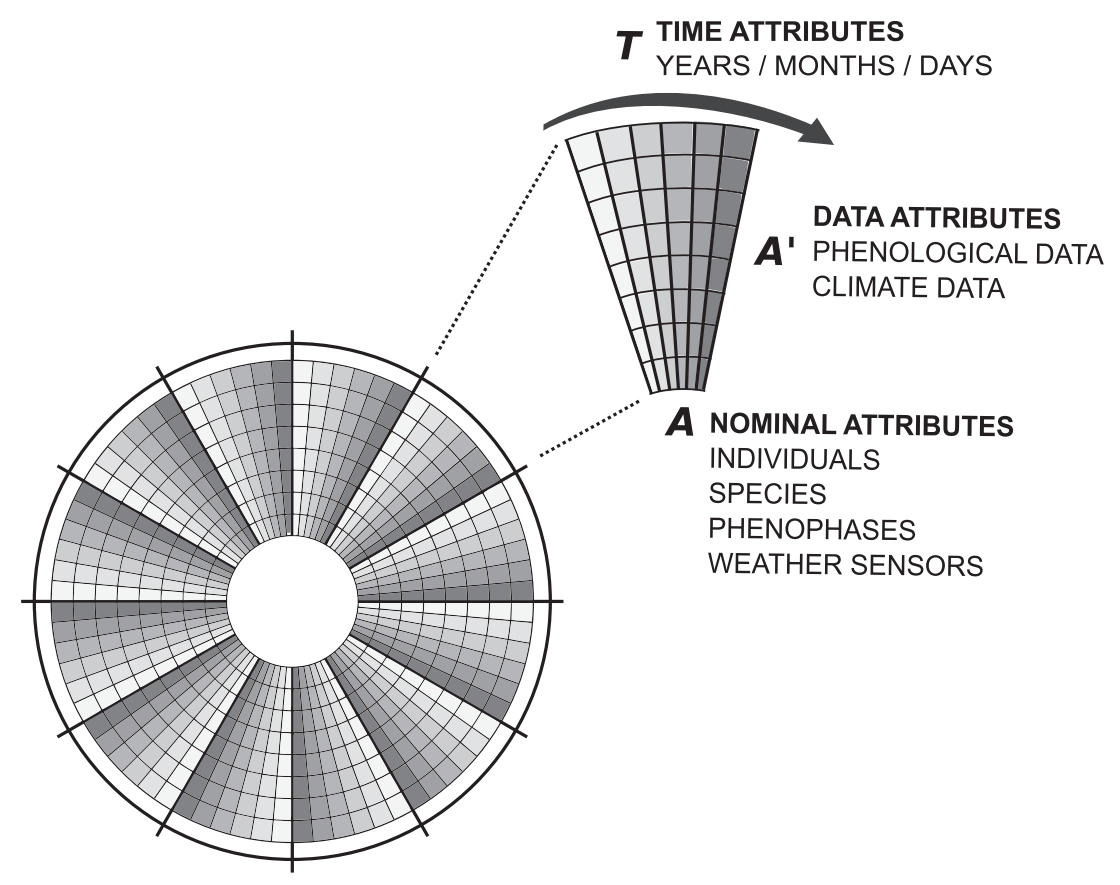

Fig. 7. Overview of the visualization proposed for numerical data.

time instants $t \in T(1 \leq t \leq n)$. With each segment $s$, we may associate one or more attributes, $A_{i}(1 \leq i \leq m)$, which may be related to phenological, climate, or image-related data. The amount of segments for one circle is equal to $n \times m$. We can compute the number of circles required using the same idea.

The data organization within the prototypes concerning time information works as follows. Circles are associated with the years, while segments are used to represent months. Therefore we have 12 segments that can be subdivided according to the number of attributes considered. Note that this distribution can be changed according to the user goals. The numerical attributes are associated with phenology and climate.

\section{Validation}

The validation of the proposed visual representation relies on a usercentered evaluation of prototypes, which encodes on-the-ground direct observation phenology data.

We carried out experiments with phenology experts aiming to validate the prototypes created to represent both, detailed and summarized data visualizations, on the context of phenology studies. The experiments aimed at evaluating the effectiveness of the proposed visualization prototypes using a task-oriented evaluation methodology.

\subsection{Target research questions}

The data considered in our case study refer to phenology observations conducted at our core cerrado site of e-phenology project described above, from 2005 to 2007. For each individual, researchers observe six reproductive phases, as follows: (i) flower buds, (ii) open flowers or anthesis, (iii) unripe fruits, (iv) ripe fruits, and the vegetative phases of (v) leaf fall and (vi) leaf flush or new leaves, as defined in Vogado et al. (2016). Recall that to each phenophase, in each observation, a score from 0 to 2 is assigned. The current implementation assumes that the target users, phenology experts, are interested in comparing phenological and climate data only. The climate data considered in our study refers to the precipitation observed for the same time period.

Some examples of typical research questions related to phenology studies that experts try to address using these data are:

1. Is it possible to visualize phenological data (all phenophase intensities) of one specific individual for a specific period of time, spanning several years?

2. Is it possible to visualize phenological data (all phenophase intensities) of one specific individual combined with climate data for a period of time, spanning several years?

3. Is it possible to identify the date of peak for a specific phenophase intensity for one individual?

4. Is it possible to visualize phenological data (all phenophase intensities) for one species, spanning several years?

5. Is it possible to visualize phenological data (all phenophase intensities) of one species combined with climate data for a period of time, spanning several years?

6. Is it possible to identify the date of peak of phenophase intensities for one species, spanning several years?

These questions aim to support the understanding of phenological changes over time and are extremely important in the context of assessing triggers and the impact of climate changes on plant phenology.

\subsection{Evaluated prototypes}

Based on the research questions presented in Section 6.1, we developed a generic visual representation using the concept of radial display and visual rhythm. The use of this representation is dependent on the kind of question being considered. Two scenarios are studied:

- Detailed visualization: questions that are related to the visualization of individual-related data (questions 1 to 3);

- Summarized visualization: questions that are related to the visualization of species-related data (questions 4 to 6).

\subsubsection{Detailed visualization}

In order to address questions from 1 to 3 presented in Section 6.1, we have implemented seven prototypes (see Figs. 8 and 9). All the prototypes present the phenophases as attributes distributed within the segments. The phenophases are presented using values 0,1 , or 2 , which 
Prototype 1

Phenological data and seasons (wet/dry)

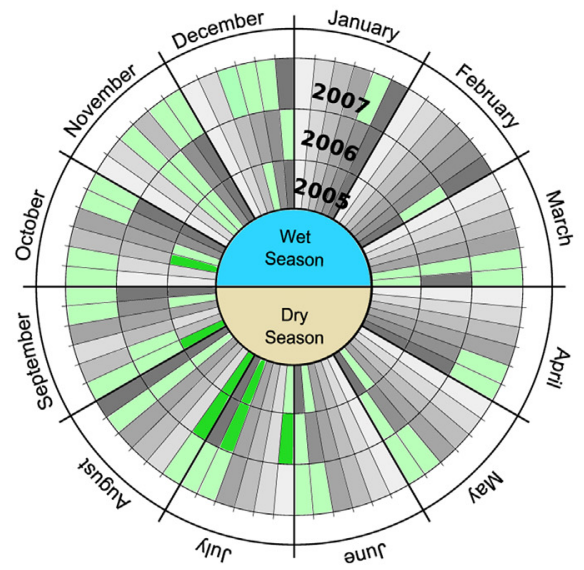

(a)

Prototype 3

Phenophases represented by icons

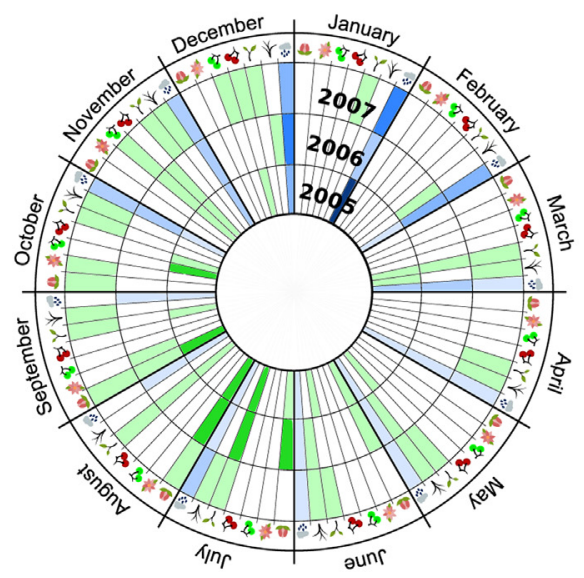

(c)

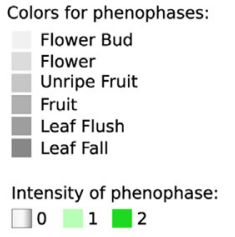

Icons for phenophases:

a. Flower Bud

Flower

Fruit

Leaf Flush

if Leaf Fall

\begin{tabular}{lll|l}
$0 \quad 1$ & 2
\end{tabular}

Variation of Precipation

: $600(\mathrm{~mm})$
Intensity of phenophase:

Prototype 5

Phenophases represented by coloured letters

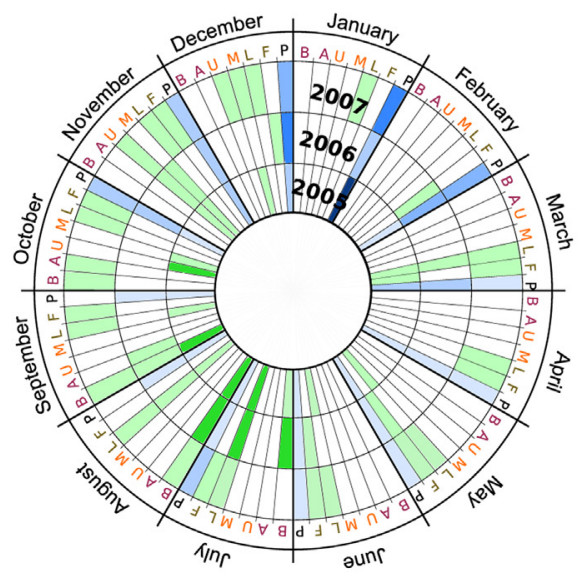

(e)
Colors for phenophases:

B Flower Bud

A Flower Open or Anthesis

$\cup$ Unripe Fruit

M Mature Fruit

$\llcorner$ Leaf Flush

$F$ Leaf Fall

Intensity of phenophase

\begin{tabular}{lll|l}
0 & 1 & 2
\end{tabular}

Variation of Precipitation (P):

$0 \quad 600(\mathrm{~mm})$
Prototype 2

Phenophases and climate data represented in colour scale

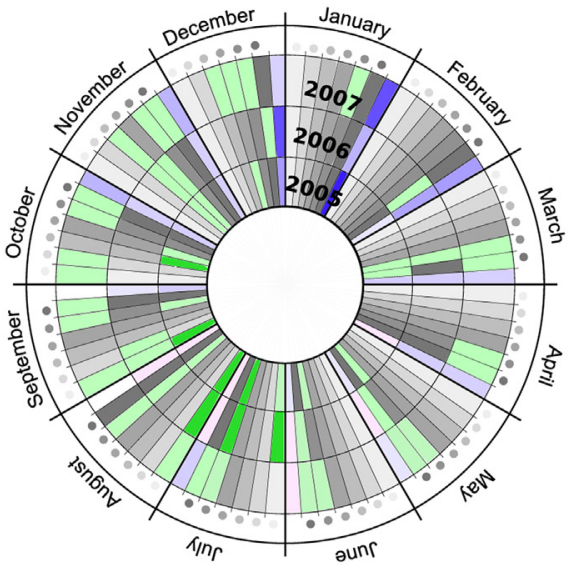

Colors for phenophases:

Flower Bud

Flower

Fruit

Leaf Flush

Leaf Fall

Intensity of phenophase:

$\square 0 \quad 1 \quad 2$

Variation of Precipation:

$0 \quad 600(\mathrm{~mm})$

(b)
Prototype 4

Phenophases represented by letters

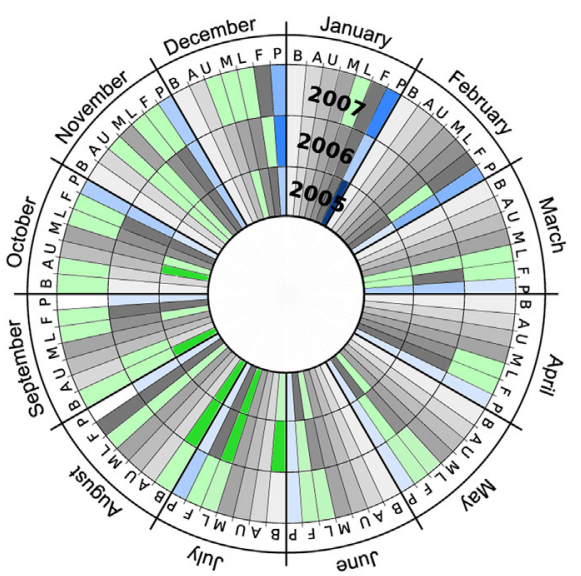

Colors for phenophases:

B Flower Bud

A Flower Open or Anthesis

U Unripe Fruit

M Mature Fruit

L Leaf Flush

Intensity of phenophase

\begin{tabular}{lll|l}
$\square$ & 1 & 2
\end{tabular}

Variation of Precipitation (P): $0 \quad 600(\mathrm{~mm})$ (d)

Prototype 6

Phenophases represented by numbers

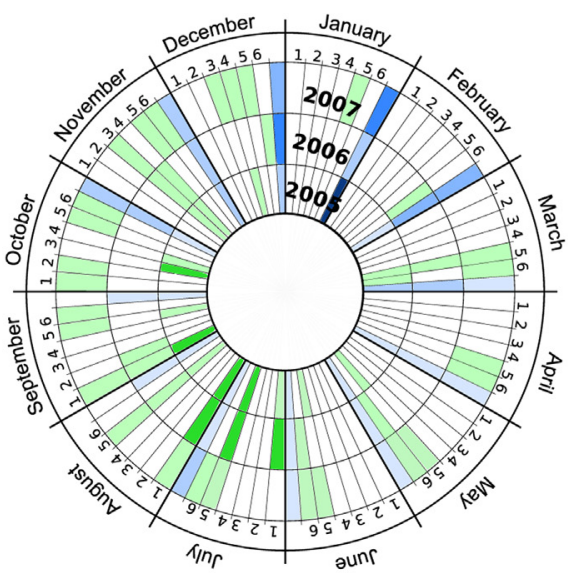

Colors for phenophases:

1 Flower Bud

2 Flower Open or Anthesis

3 Unripe Fruit

4 Mature Frui

5 Leaf Flush

Intensity of phenophase

\begin{tabular}{lll|l}
$\square$ & 1 & 2
\end{tabular}

Variation of Precipitation $(\mathrm{P})$

$0 \quad 600(\mathrm{~mm})$

(f)

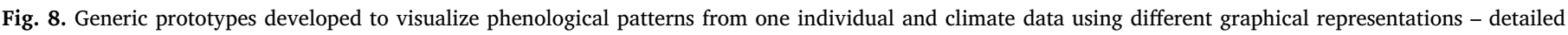
visualization level. 


\section{A) Rainfall}

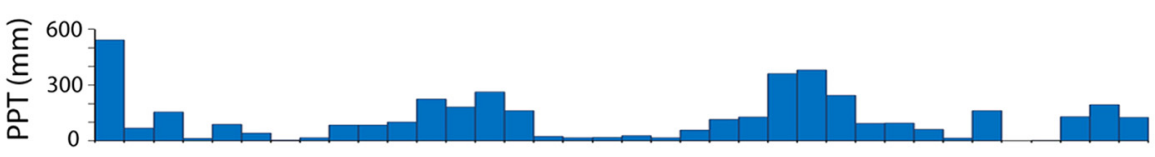

\section{B) Vegetative phenophases}

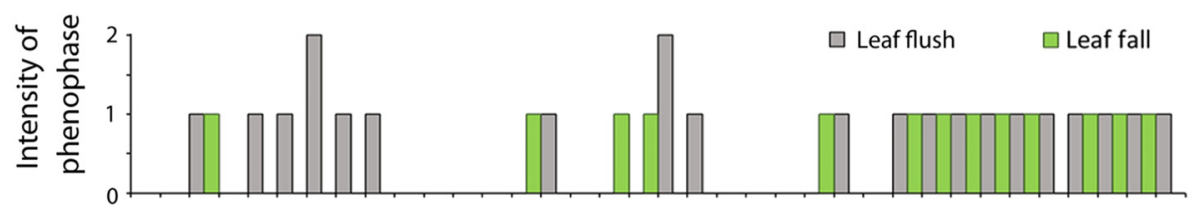

\section{C) Reproductive phenophases}

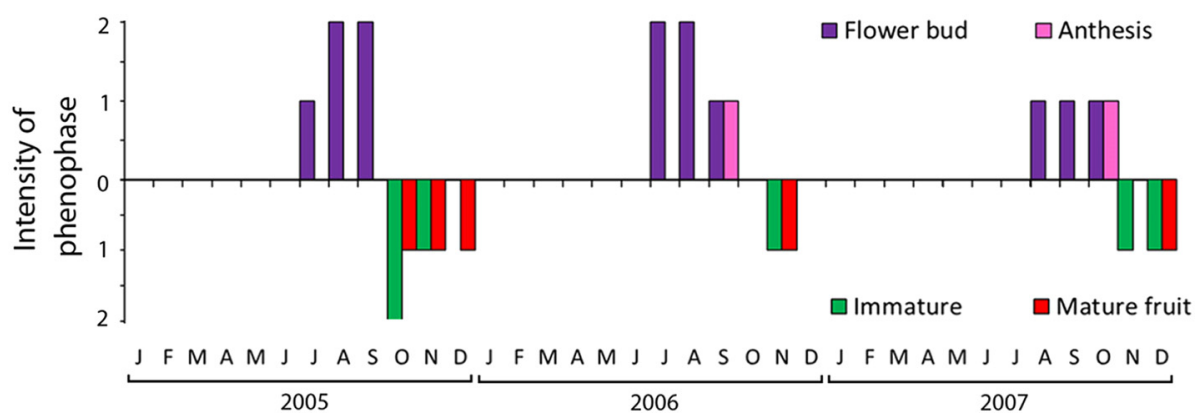

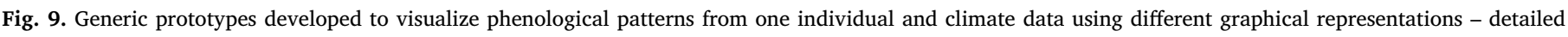
visualization level. Example using liner graph.

represent the respective observed intensity.

The main difference between them is concerned with how the climate data is shown and, in the case of Prototype 7, how the phenophases and intensities are represented - in this case, in a linear way (Fig. 9 - Prototype 7). This is the most common method used by experts and therefore considered here as our baseline. The prototypes whose evaluation is close to the evaluation of the baselines will be considered good candidates for being used in phenology studies.

In the first prototype (Fig. 8 - Prototype 1), the climate data are shown in the more inner circle in a generic fashion. In all other prototypes (Fig. 8 - Prototypes 2, 3, 4, 5, and 6), in turn, climate data are placed along with the phenophase data (regions highlighted in blue).

In all prototypes, we consider for each month that the experts want to visualize all six phenophases. Furthermore, note that in the first, second, and fourth prototype, we represent the phenophase intensity values equal to 0 in gray, while in the other prototypes, in white. We also change the way a phenophase is represented: gray scale (Fig. 8 Prototype 2), icons (Fig. 8 - Prototype 3), letters (Fig. 8 - Prototypes 4 and 5), and numbers (Fig. 8 - Prototype 6). With these seven representations, we want to support the visualization of cyclical data with the objective of allowing the identification of patterns and relations among the different variables. In the considered examples, relations among phenophase intensities and precipitation are considered. All prototypes also allow the identification of peaks in the intensity values (bright green cells).

\subsubsection{Summarized visualization}

With regard to questions from 4 to 6 presented in Section 6.1, five other prototypes were designed. All prototypes implemented for this step (see Figs. 10 and 11) consider data about one single species. We are using data about the species Myrcia guianensis (Vogado et al., 2016).
The main difference here is that data about one species include records associated with one or more individuals, which demands the use a summarization approach. In this case, we applied our approach based on the visual rhythm representation. To determine the phenophase data to be presented, we computed the visual rhythm using an aggregation function. Thus, instead of presenting values of intensity, here we calculate how many individuals of a particular species present the phenophase value different from zero. For instance, for phenophase flower bud, we count how many individuals presented 1 or 2 in the time period considered in the study. Therefore, the values associated with phenophase segments that we present in our visualization refer to the relative percentage of individuals with phenophase intensity value different from zero or just the presence of phenological activity. Similarly to the prototypes of individuals, all prototypes have six phenophases and the fifth prototype is our baseline linear visualization (Fig. 10 - Prototype 5). Besides that, to identify each phenophase, we use different strategies: gray-level scale (Fig. 10 - Prototype 1), icons (Fig. 10 - Prototype 2), letters (Fig. 10 - Prototype 3), and numbers (Fig. 10 - Prototype 4).

\subsection{Experimental setup}

\subsubsection{User profiles}

Eleven phenology experts were recruited by email to take part in the evaluation of the prototypes - six active researchers (Ph.D.), five Ph.D. candidates. One of the Ph.D. candidates did not fill out the evaluation form related to prototypes of species. The key selection requirement relied on their expertise in plant phenology domain. Subjects of any age (over 18 years) or gender were accepted in the study. 
Prototype 1

Phenological data represented in colour scale and seasons (wet/dry)

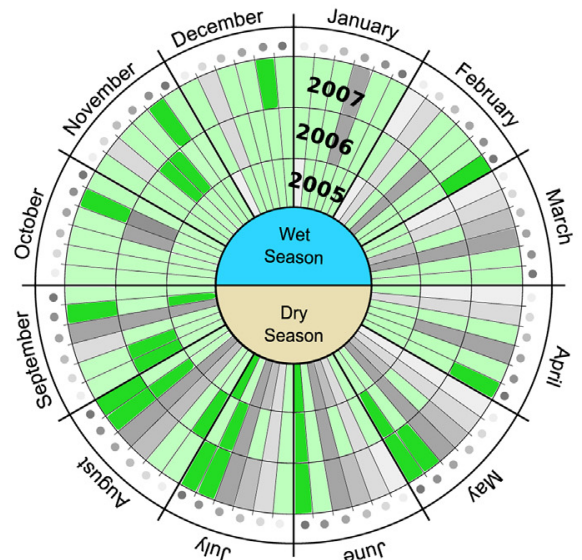

(a)

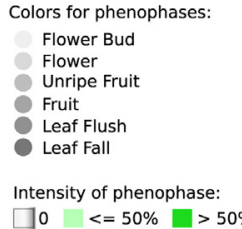

$\square 0 \quad<=50 \% \square>50 \%$

Letters for phenophases: B Flower Bud A Flower Open or Anthesis U Unripe Fruit

M Fruit

L Leaf Flush

F Leaf Fall

Intensity of phenophase

$\square 0<=50 \% \quad>50 \%$

Variation of Precipitation $(\mathrm{P})$ :

$0 \quad 600(\mathrm{~mm})$ (c)
Prototype 2

Phenophases represented by icons
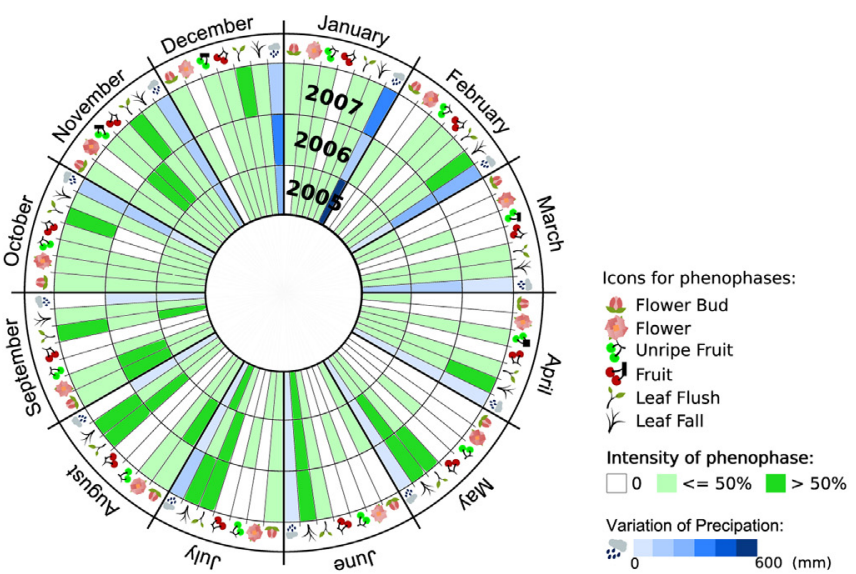

(b)

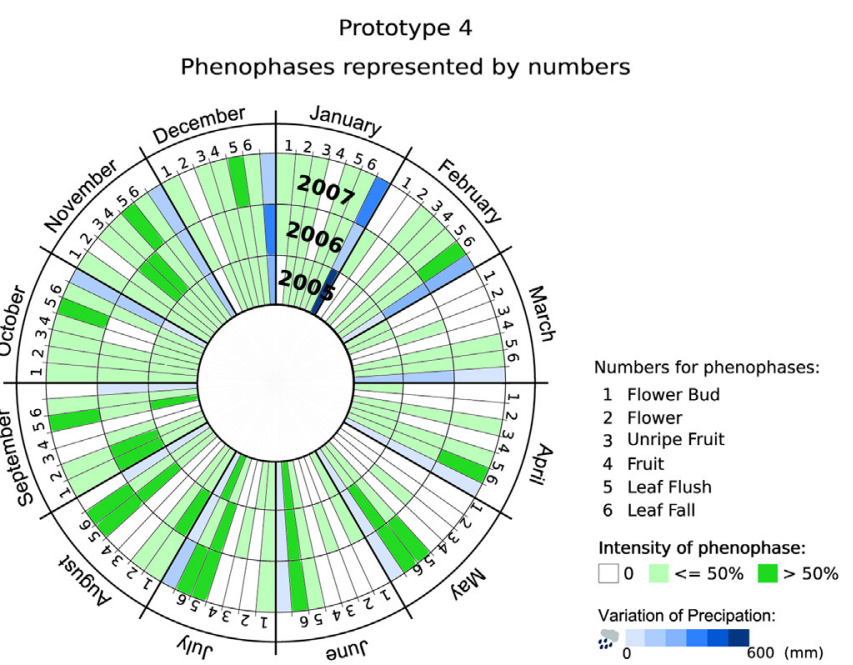

(d)

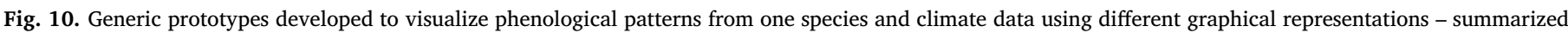
visualization level.

\subsubsection{Task}

Before any procedures the research plan and forms were submitted for approval in the Unicamp humans ethical committee. We created two types of forms - one used to evaluate prototypes representing individuals data and another to evaluate those that manage species data. In both forms, the evaluators were asked to identify peaks of flowering and fruiting in specific dates and if they could identify a relationship between leaf flush and precipitation.

\subsubsection{Procedure}

All evaluators were asked to evaluate all prototypes.

Each prototype was associated with a different evaluation form. All recruited evaluators filled out the form about individuals' prototypes and only ten filled out the form about species' prototypes. For each question of the forms, the user should indicate, in a Likert scale (from 1 to 5), whether it was easy to identify the required standards on issues. In the scale: 1 means that the user totally disagrees; 2, disagrees; 3 , neither agrees or disagrees; 4 , agrees; 5 , totally agrees.

\subsubsection{Opening questionnaire}

Evaluators were asked to fill out a questionnaire concerning their familiarity with computers and visualization techniques, as well as, their expertise with tools to visualize phenological data. Only one participant indicated to know the information visualization area and reported to have used the package ggplot2 from language $\mathrm{R}$ to represent data of interest.

\subsection{Results}

Fig. 12 shows the results with regard to the satisfaction of users concerning how easy is the identification of phenophase intensities over years considering all prototypes evaluated. Fig. 12a shows the results of prototypes that represent phenological data at detailed level (Prototypes $1,2,3,4,5,6$, and 7 from Figs. 8 and 9), i.e. using data observations about individuals, while Fig. $12 \mathrm{~b}$ shows the results of prototypes that represent phenological data in a summarized level (Prototypes 1, 2, 3, 4, and 5 from Figs. 10 and 11), i.e., using data from species. In general, higher scores were observed for Prototype 3 related to data of individuals, i.e., this is the prototype whose evaluation is 


\section{A) Rainfall}

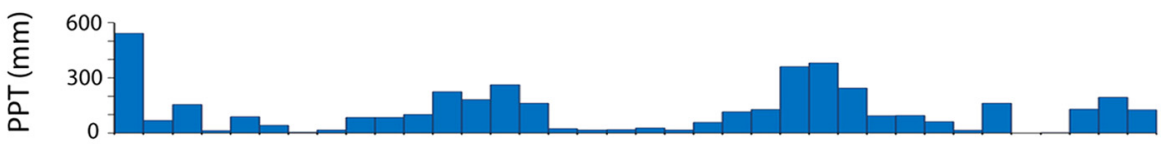

\section{B) Vegetative phenophases}

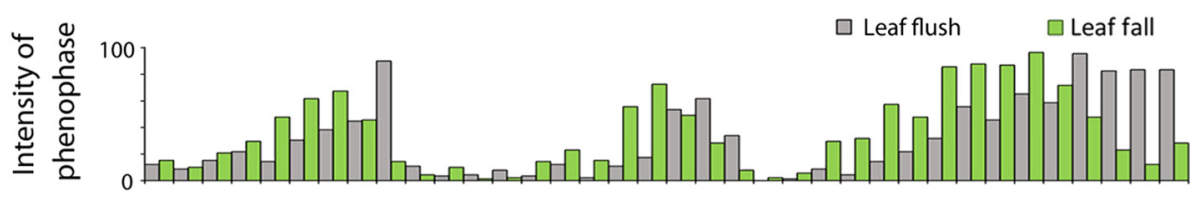

\section{C) Reproductive phenophases}

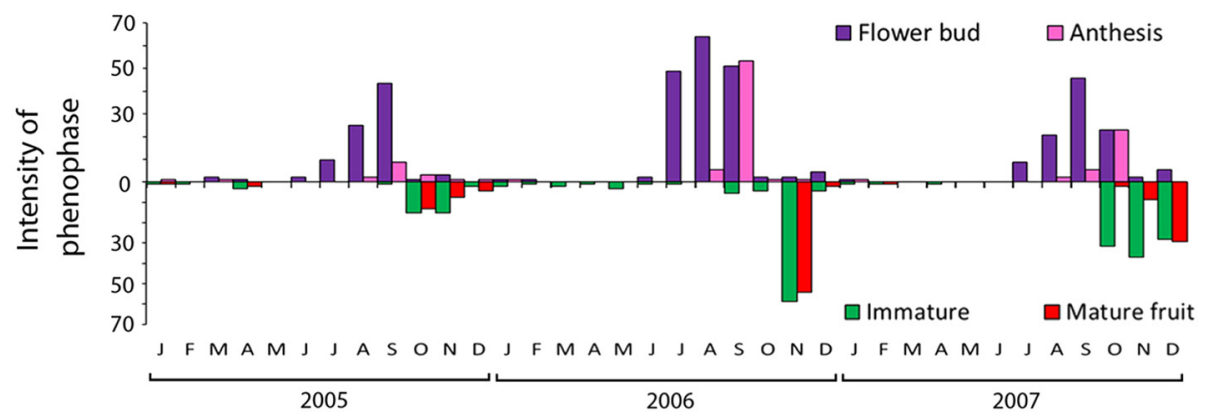

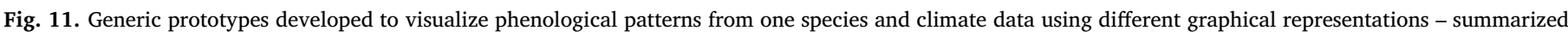
visualization level. Example using linear graph.

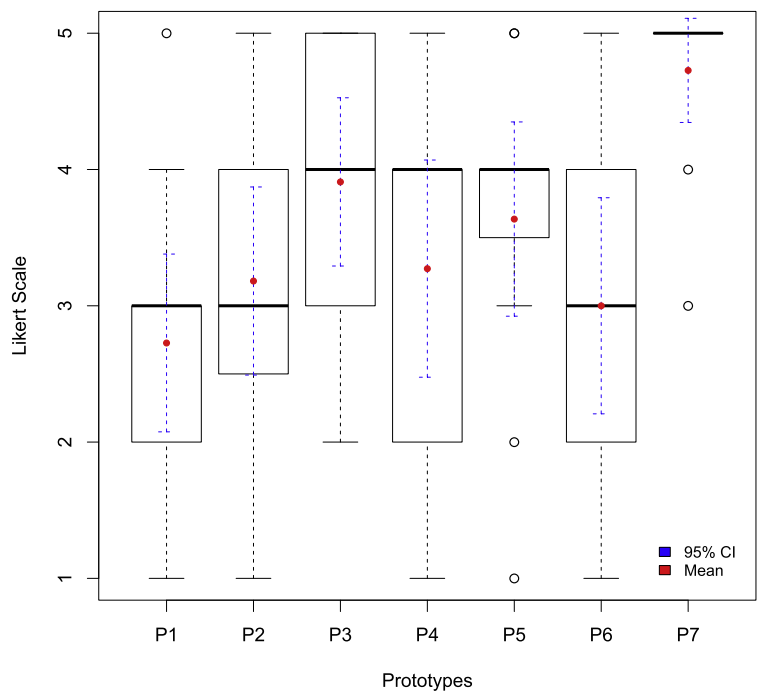

(a) Prototypes for individuals-related data

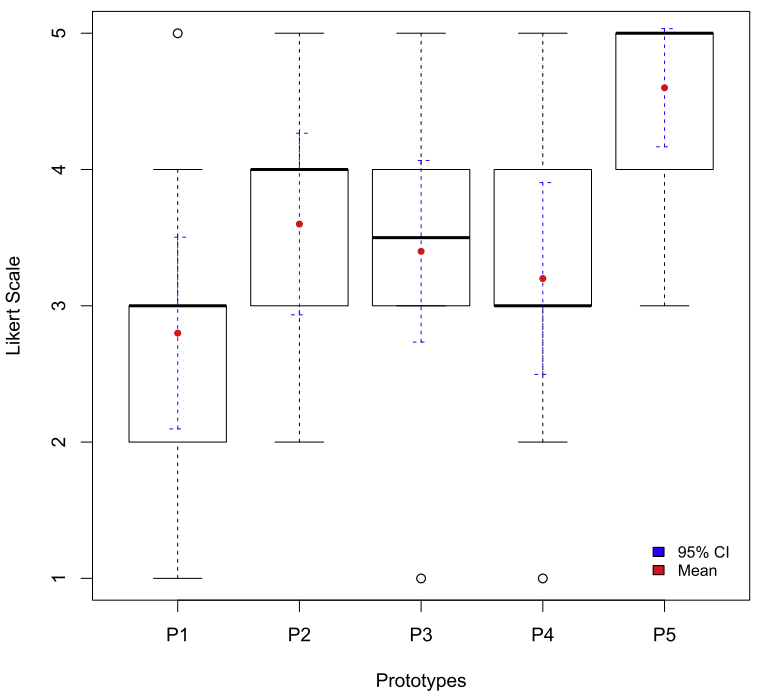

(b) Prototypes for species-related data

Fig. 12. How easy is the identification of phenophase intensities over time.

closer to the baseline (Prototype 7 for individuals). Regarding species data (Fig. 12b), Prototypes 2, 3, and 4 have similar results. As it was expected, in all situations the baseline has the best scores, due to the familiarity of phenology experts in using the data representations employed by them.

Fig. 13 shows the results with regard to the satisfaction of users concerning how easy is the task of relating the production of flowers with the production of flower bud. In this case, we aim to assess whether it was understandable and easy for users to relate two phenophases. There is no clear winner with regard to prototypes for individuals (Fig. 13a), but for the baseline. Prototype 3 (for individuals) has the highest average score. All the prototypes for species data yielded similar results (Fig. 13b), Prototype 3 with a slightly better mean.

Fig. 14 shows the results with regard to the satisfaction of users concerning how easy is the identification of peaks of flowering for both 


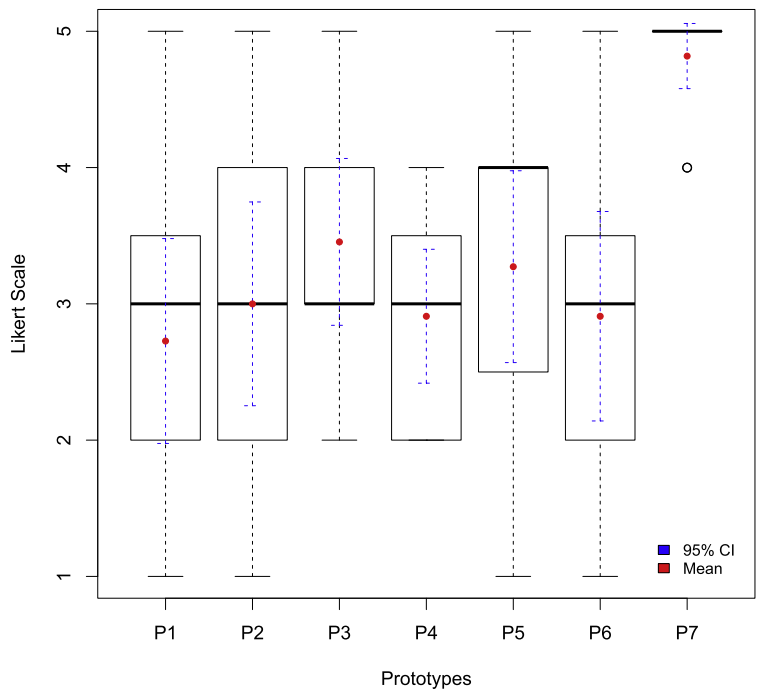

(a) Prototypes for individuals-related data

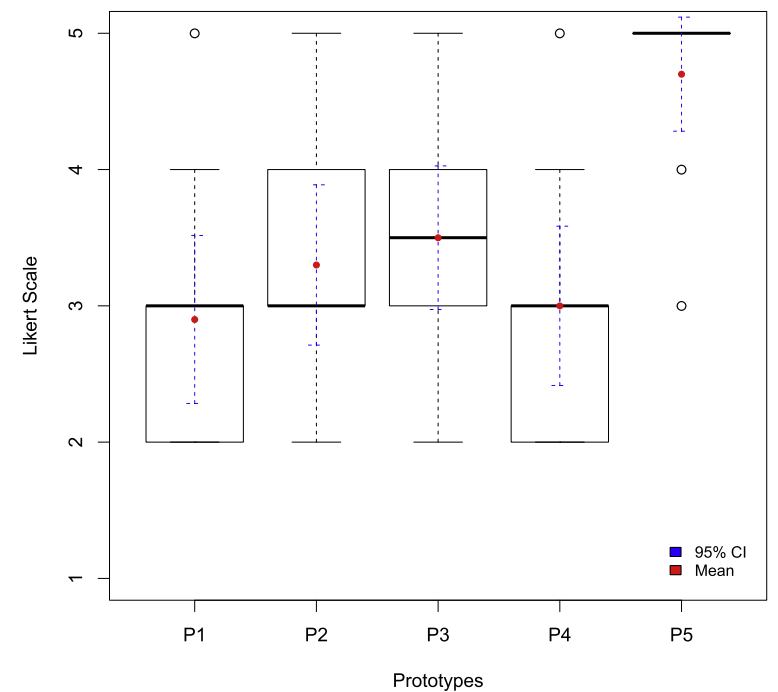

(b) Prototypes for species-related data

Fig. 13. How easy is the identification of the relation between the production of flowers and the production of flower bud.

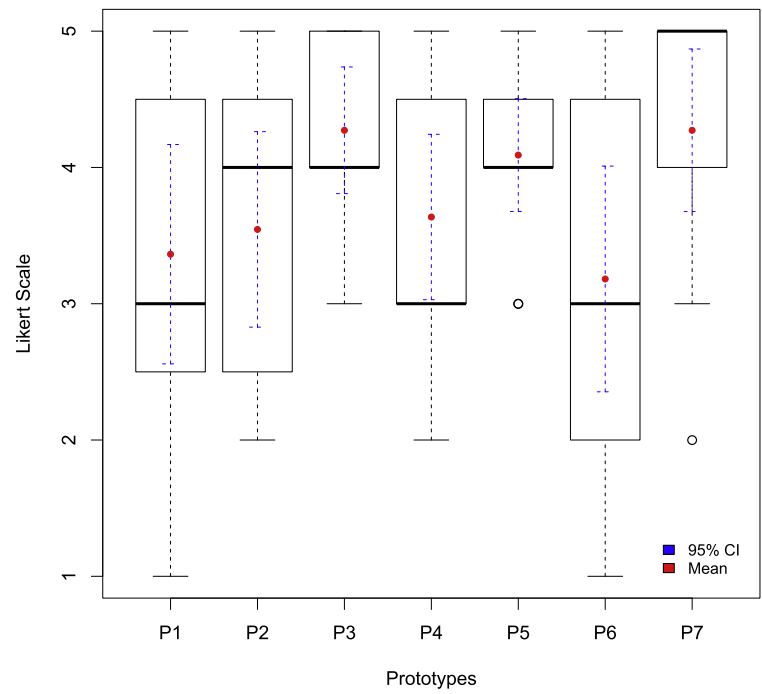

(a) Prototypes for individuals-related data

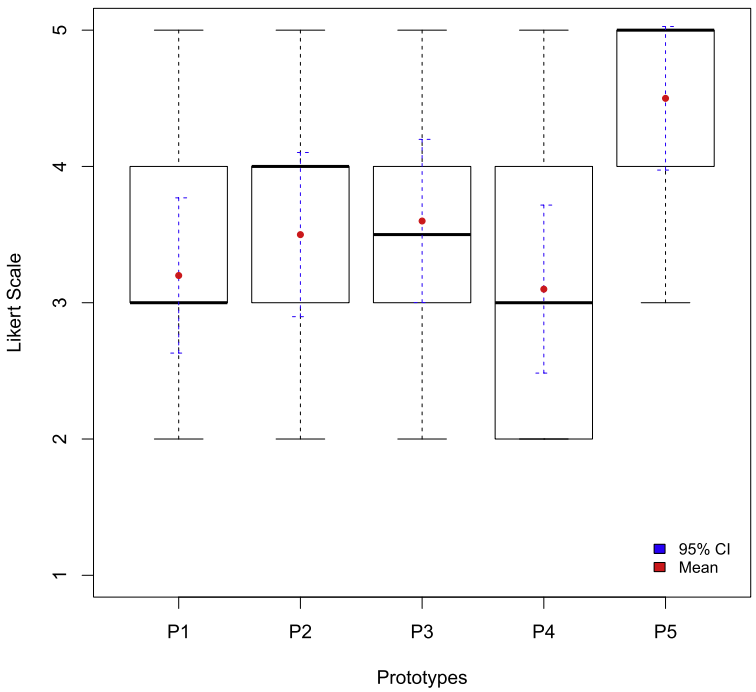

(b) Prototypes for species-related data

Fig. 14. How easy is the identification of flowering.

individuals and species. Here, we aim to assess whether the users are able to identify when flowering occurs. Again, Prototype 3 for individuals (Fig. 14a) and Prototypes 2 and 3 for species (Fig. 14b) were those which had the best scores.

Fig. 15 shows the results with regard to the satisfaction of users concerning how easy is the identification of peaks of fruiting for both individuals and species. Here, we aim to assess whether the users are able to identify when fruiting occurs. Again, Prototype 3 for individuals (Fig. 15a) and Prototypes 2 and 3 (Fig. 15b) for species were those which had the best scores.

Finally, we investigated how easy is for users to correlate climatic data (rainfall) with leaf flush phenophase. Fig. 16 shows the results. In this task, no prototype achieved outstanding scores. Even the baseline for individual data (Prototype 7, Fig. 16a) was not well evaluated. A slightly better mean is observed for Prototype 3 when dealing with individual's data. These results demonstrate how challenging is the task of providing appropriate visualization approaches for supporting the identification of correlation among complex variables.

The evaluators also provided their feedback upon all prototypes in a text field. We have selected the most relevant positive and negative comments provided. In general, the evaluators commented that the use of a radial structure is a good strategy for displaying multivariate temporal data into a single representation. They believe, however, that at the same time, too much information is encoded in the representation, which hampers the analysis process. For example, in several studies, they are not interested in analyzing all phenophases altogether. Usually studies focus on specific stages of the plant life cycle (e.g., the reproductive phenology related to the flower bud and flower phenophases). One suitable alternative to address this issue would be the support of the use of filtering options (e.g., based on years, phenophases, or data types) with which users may select and analyze more easily data of interest.

The evaluators believe that the approach of encoding information about wet and dry seasons in the inner ring as used in Prototype 1 (for both species and individuals) is a suitable alternative to analyze the impact of environmental conditions on plants. Except for Prototype 1, climate data is encoded as a variable, which was welcome by the evaluators. On the other side, the evaluators commented that this strategy makes difficult to establish correlations among climate and phenological data. In some situations (e.g., Prototypes 4 and 5 for species), the 


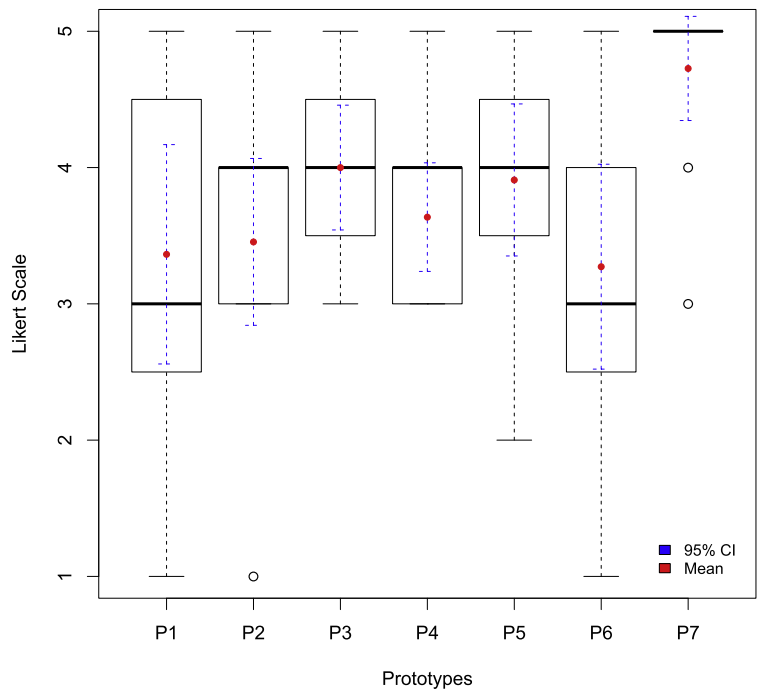

(a) Prototypes for individuals-related data

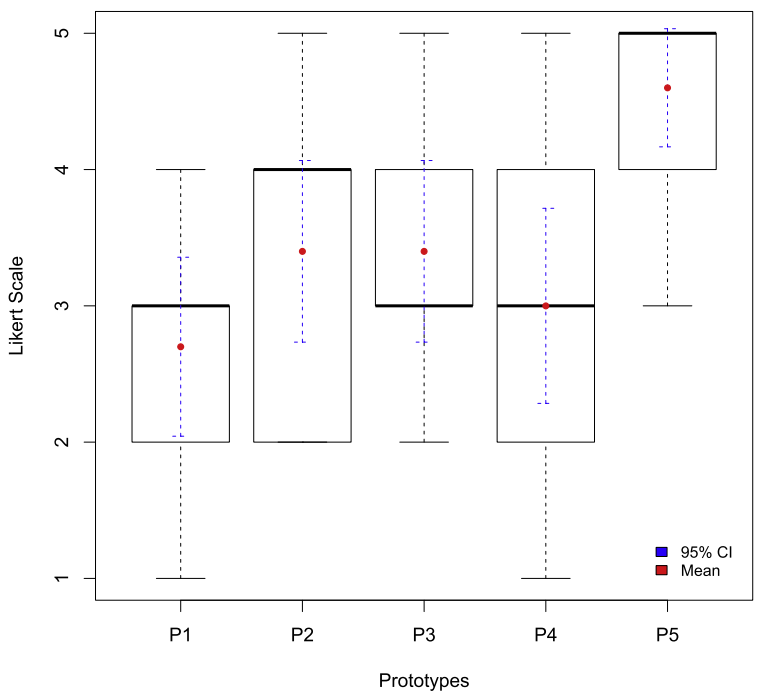

(b) Prototypes for species-related data

Fig. 15. How easy is the identification of fruiting.

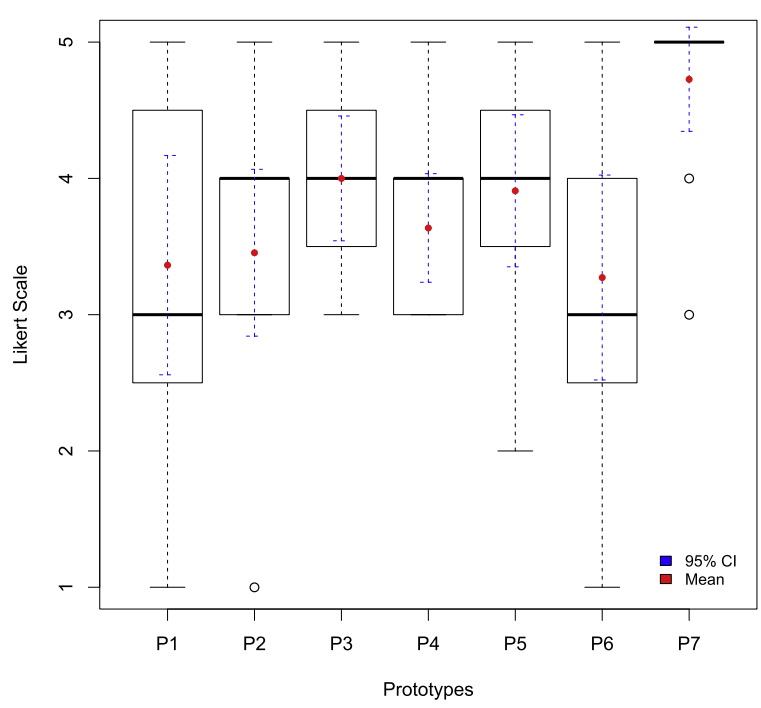

(a) Prototypes for individuals-related data

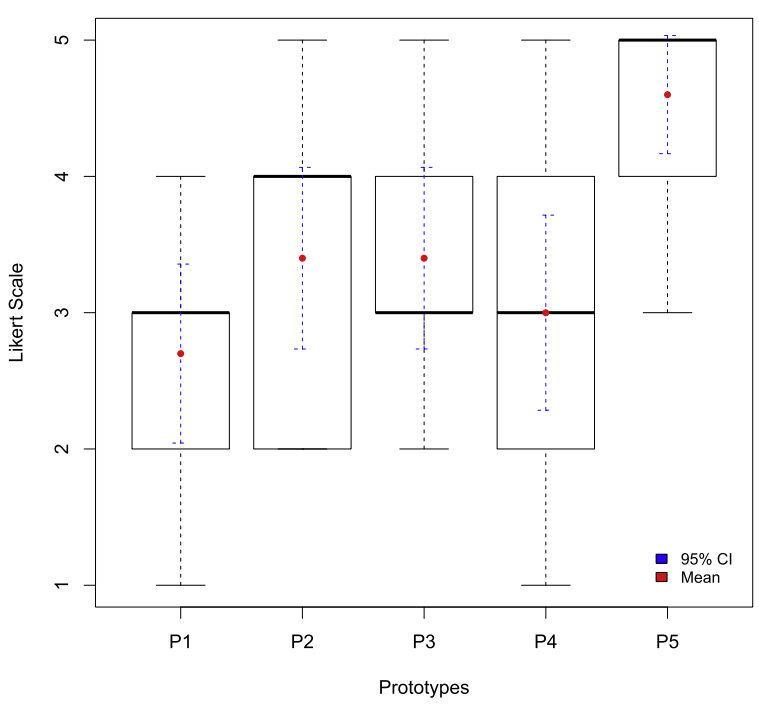

(b) Prototypes for species-related data

Fig. 16. How easy is the identification of impact of rainfall to leaf flush.

provided color information made the correlation identification process even more difficult. Allowing users to change the type of chart utilized for represent climatic data based on their specific interests would address this issue.

The different prototypes use different strategies to support the identification of phenophases. For example, for individuals (see Fig. 8), it is used gray-level colors (Prototypes 1 and 2), icons (Prototype 3), letters (Prototypes 4 and 5), and numbers (Prototype 6). The evaluators, in general, preferred letters to numbers, and icons to letters. They believe, however, that the used icon-based representation should be avoided in scientific reports. To facilitate the identification of years or phenophases, customized controls for highlighting specific rings, segments, or cells could be provided.

Furthermore, prototypes (e.g., 3, 5, and 6 for individuals) with white background (usually used to encode phenophases with intensity equal to zero) are preferred to the ones with gray-level colors, because the evaluators were able to identify more easily the intensity and the peak of phenophases. We believe that the existence of customized controls to support the definition of different colors for specific data (segments) would be useful.
Another positive aspect of the radial structure emphasized by evaluators refers to the possibility of presenting data about multiple years into a single representation. In fact, this is the most commented drawback of the baselines (Prototype 7 of individuals and Prototype 5 for species). On the other side, according to the evaluators, the inner segments, which refer to data observed in year 2005, are too small. One alternative to address this issue would be the existence of customized controls for zooming in and zooming out (overview and detail levels). This approach would be useful also in the context of the use of the radial structures for representing long-term (multiple years) temporal data.

\subsection{Discussion}

Phenology studies rely on the analysis of the life cycle of living beings and its relationship with weather variables. Usually, performed analyses rely on the use of different kinds of data, typically handled through multiple tools. Therefore, scientists concerned with phenology studies usually seek support from a large set of information systems. This, of course, brings about all kinds of interoperability problems due 


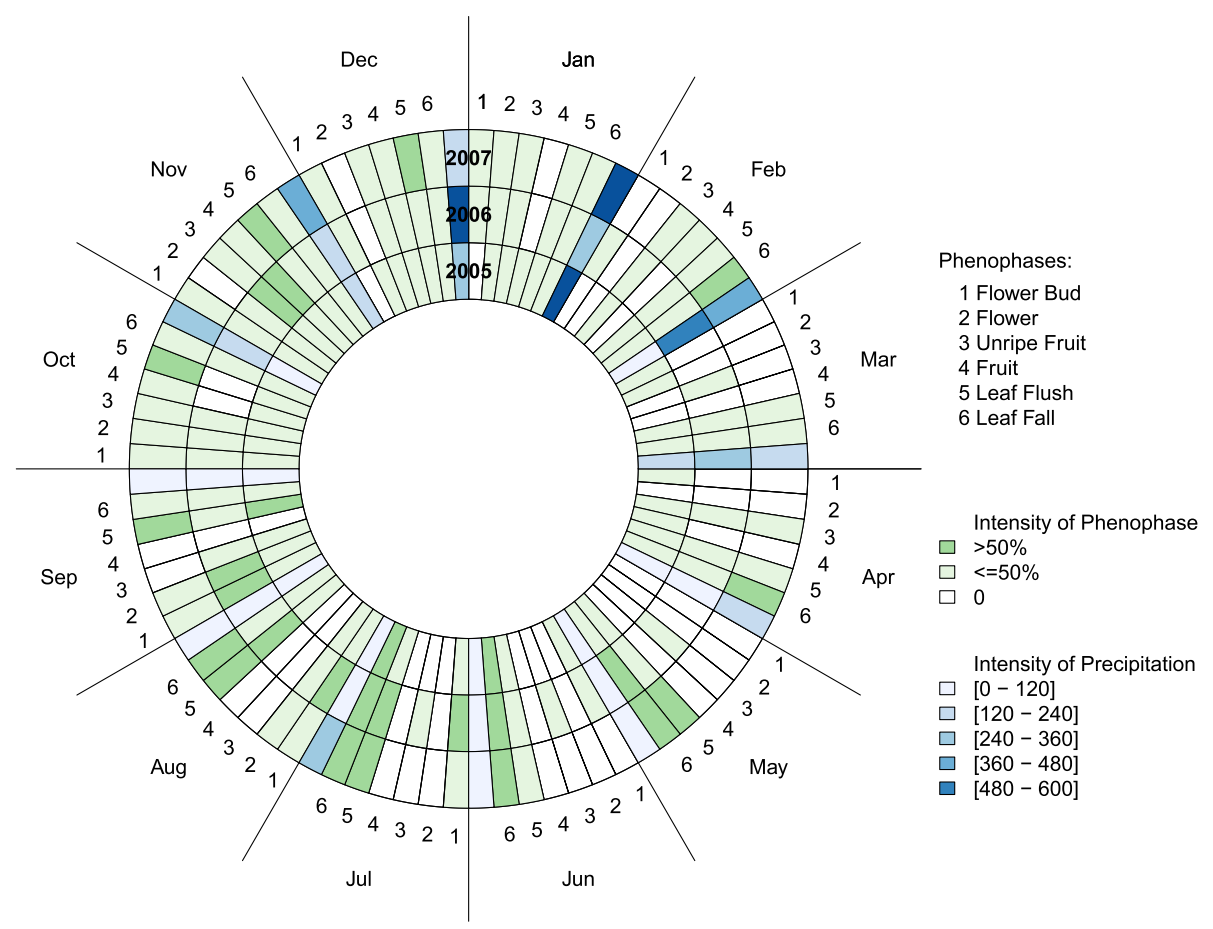

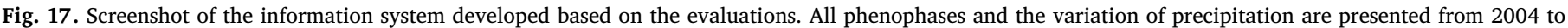
2007 (same data showed for Prototype 4 at summarization level).

to system mismatch, data diversity, and variety of user profiles (Mariano et al., 2016b). Our approach is based on the possibility of using one single technique to visualize and manage different kinds of data, including multiple phenophases and environmental information in a comparative way, an especially important situation where a large number of temporal data has to be analyzed.

In the performed evaluations, we wanted to investigate what is the perception of users about visualizing multiple variables at the same time. In our evaluation, we opted for interface mockups associated with worst-case scenarios for the visualization. In this context, we presented data about all phenophases at the same time with the goal of assessing if, for the possible users, it would be possible to identify correlations between plant phenophases and climate data, and also to identify useful information regarding the whole life cycle of individuals or species. Note also that the considered scenarios, involving multiple phenophases, are useful for intraspecific comparison of individuals of the same species (Soares and Morellato, 2018; Vogado et al., 2016; Mariano et al., 2017).

In summary, Prototype 3 for individuals and Prototypes 2 and 3 for species were the most promising ones to be used to support complex phenology studies, mainly related to the analysis of multidimensional data over several years. The use of icons as exploited in Prototype 3 (for individuals) and Prototype 2 (for species) seems more appropriate for data presentation in non-scientific purposes or in scenarios involving less experienced users (such those related to citizen science actions).

Also, the performed evaluation suggests the need of the implementation of interaction mechanisms. We noticed that the users would perform the proposed tasks more easily, possibly with the assistance of customization tools (e.g., interaction facilities such as selection or filtering features) to reduce and increase the amount of data presented in the proposed visual structure according to user convenience. In particular, we noticed that these kinds of interactions are important mainly when phenology experts want to compare individuals from the same species or individuals from the same species but from different locations.

In fact, from the lessons learned from this study, we have conceived and have been implementing a complete visualization information system where the user can interact and control what the users will see and explore from the existing datasets. For example, users may select the number of phenophases and the color with which phenological data will be presented. Some screenshots are presented below.

Recall Prototype 4 (see Fig. 10d), which was used to depict the pheno-phase intensity for one species during the years of 2005, 2006, and 2007. In Fig. 17, we present the screenshot of the information system being conceived to represent the same data considered in Prototype 4. Suppose now that the user of the system is interested in studying only the reproductive cycle of individuals of this species. In this case, the user may select phenophases flower bud, flower, unripe fruit, and fruit, indicating the interest in visualizing their intensity scores. Fig. 18a presents the radial structure layout after the selection of reproduction-related phenophases. Similarly, the user may be interested in comparing the intensity scores only for two phenophases. Fig. 18b shows an example using the radial layout structure after the selection of only flowering-related phenophases (e.g., flower and flower bud).

In this tool, both naive and experienced users will be able to visualize collections of temporal multidimensional data. More experienced users may be interested in narrowing down data analysis, by taking advantage of selection and filtering options available in the tool interface. By using those features, the user may, for example, select specific phenophases (e.g., those related to reproductive cycle as illustrated before), individuals (e.g., those of a single region), and years (e.g., aiming to identify changes over specific time periods). Also, customization tools may be used, for example, to select and define different colors to represent the intensity of particular phenophase.

Another usage scenario refers to the possibility of using the proposed visualization approach for depicting monthly data related to vegetation indices extracted from sequences of images (Alberton et al., 2017, 2014). In this case, the daily changes can be observed and compared for different individuals (represented as regions within the image) of interest. Figs. 19 presents an example of daily image taken by digital cameras associated with a region of interest. Using the region of interest, we can process the daily images to obtain the temporal features, such as the gcc variation (Alberton et al., 2017), which can be 


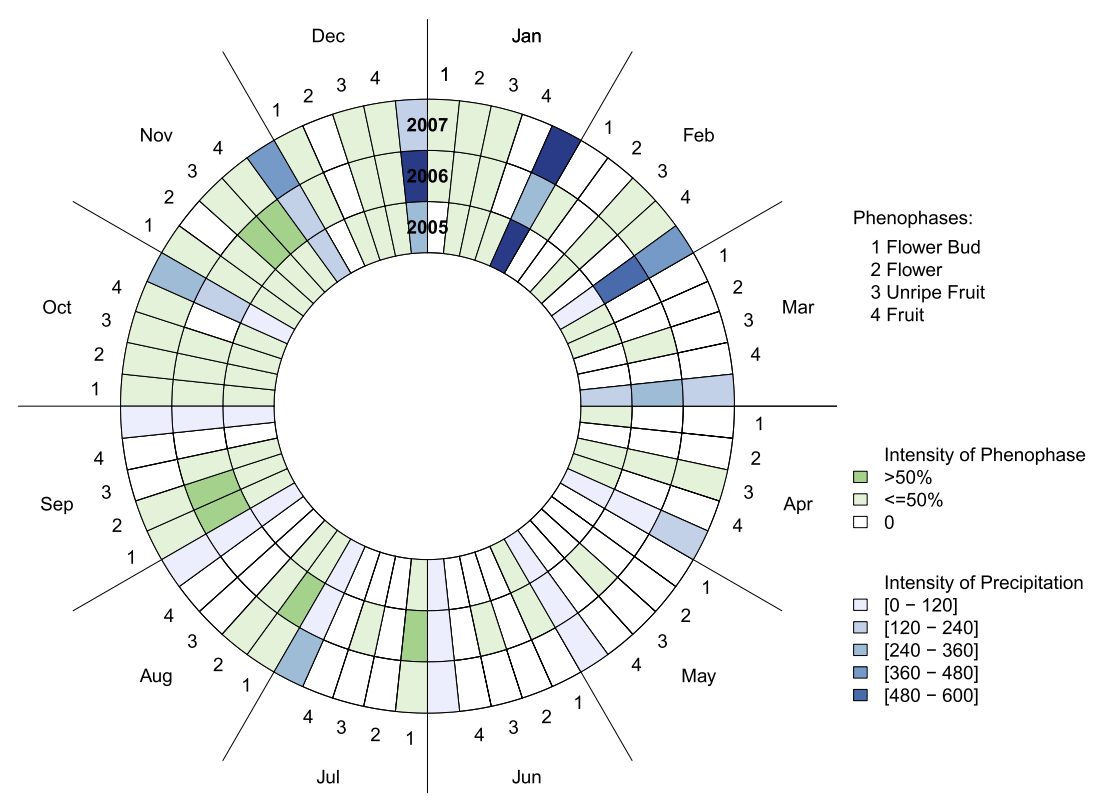

(a)

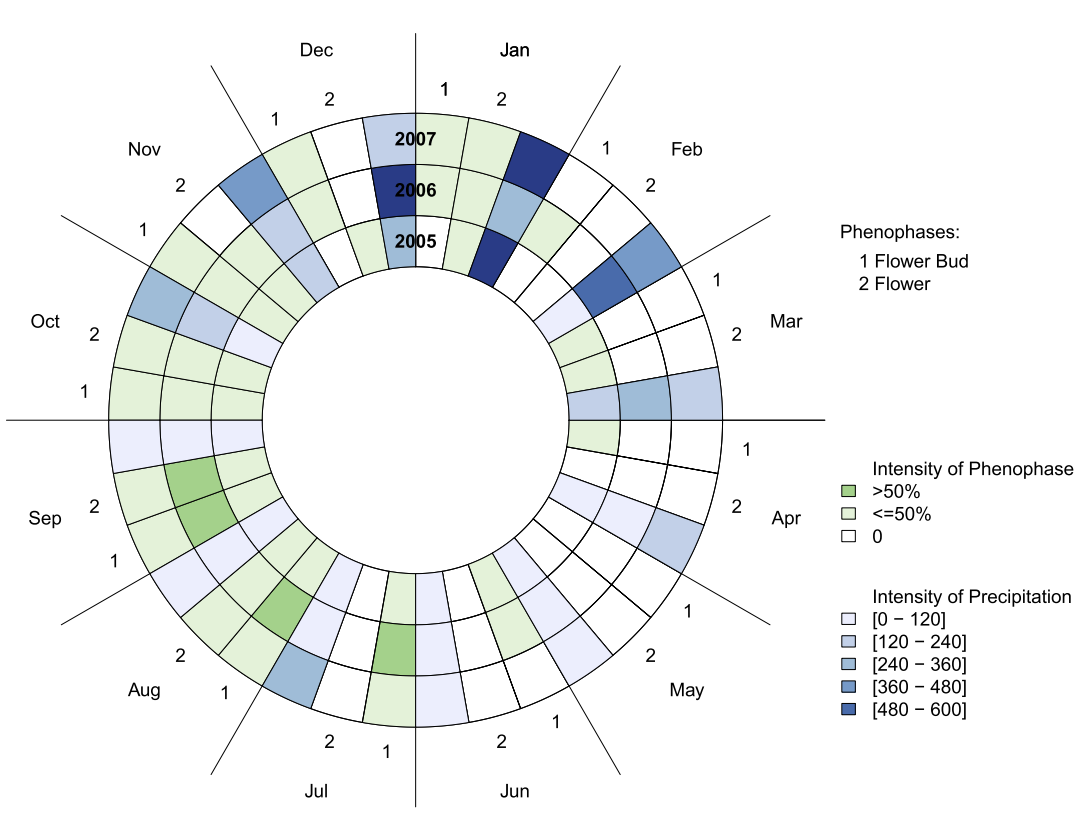

(b)

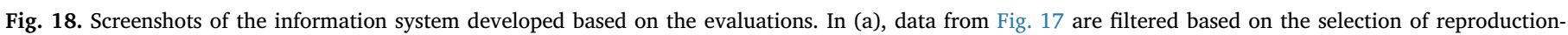
related phenophases. In (b), data from Fig. 17 are filtered based on the selection of two phenophases (flower bud and flower).

visualized using the proposed visual structure. Fig. 20 shows the results for the gcc variation over 2014, 2015, and 2016, associated with an individual of species Caryocar brasiliensis.

\section{Conclusions}

In traditional phenology studies, collected data refer to the observation of phenology made by experts in the field and usually are correlated with climate data obtained by weather sensors (Mariano et al., 2016b; Morellato et al., 2016). Since the phenology studies are based on short to long-term time series and different types of data, the experts often face the challenge of understanding relations among different data types over time.

This paper has introduced a novel visualization approach for presenting cyclical multidimensional temporal data associated with phenology studies. Our proposal combines a radial visualization with the visual rhythm approach, which are applied in numerical and climate data. The use of radial layouts aims at providing contextual information about multiple variables varying over time. The visual rhythm approach, in turn, is used to summarize large volumes of multidimensional data into a more compact and easy-to-process representation. That sort of representation has not been found on phenology studies, even on those using circular approaches (Morellato et al., 2010a).

We also discuss different usage scenarios of the proposed visualization approach, demonstrating its potential in the target application. We created 12 prototypes for on-the-ground, traditional direct phenology observation data and performed evaluations with phenology 


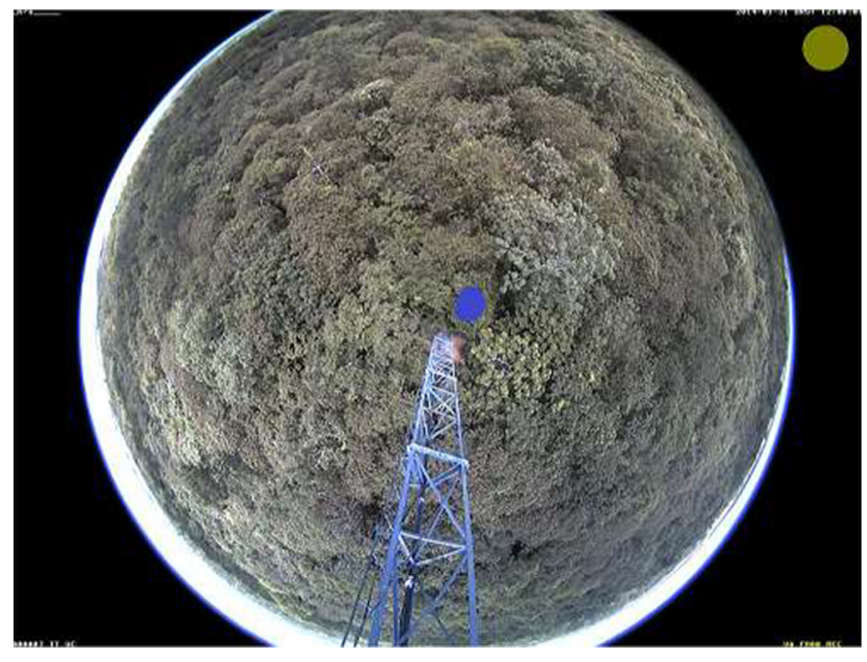

Fig. 19. Example of daily vegetation image taken in the context of e-phenology project, and the mask (blue shadow) for a species crown, that represents a region of interest (ROI). Based on the processing of the ROI within the image, we can extract some temporal features for the vegetation, for instance, the green chromatic correlation Gcc variation (Alberton et al., 2017; Alberton et al., 2014).
These data could also be displayed along with climate data such as precipitation. Future work will also be dedicated to the creation of tools based on the different prototypes considered in our study. One promising venue is to integrate the visualization possibilities with features to support circular statistic analyses (Morellato et al., 2010b). Besides that, we plan to use the prototypes to visualize the results produced by the proposed Change Frequency Heatmap approach to visualize temporal changes in phenological data (Mariano et al., 2017) and also integrate the prototypes with recently proposed tool to manage phenology data (Mariano et al., 2016b), both of them in the context of the e-phenology project.

\section{Acknowledgment}

The present work was supported by the São Paulo Research Foundation (FAPESP) thorough Microsoft-FAPESP Virtual Institute [grants \#2013/50169-1, \#2013/50155-0 and \#2014/50715-9], FAPESP [grants \#2007/59779-6, \#2009/18438-7, \#2010/51307-0, \#2014/12236-1, \#2015/24494-8, \#2016/50250-1, and \#2017/ 20945-0]. We also thank the support from CNPq and CAPES [grant \#88881.145912/2017-01]. GM received PhD fellowship from CAPES and CNPq [grant \#162312/2015-6], VGS receives a post-doc fellowship from FAPESP [grant \#2014/13889-4]. LPCM and RST receive a research productivity fellowship from CNPq (National Council for

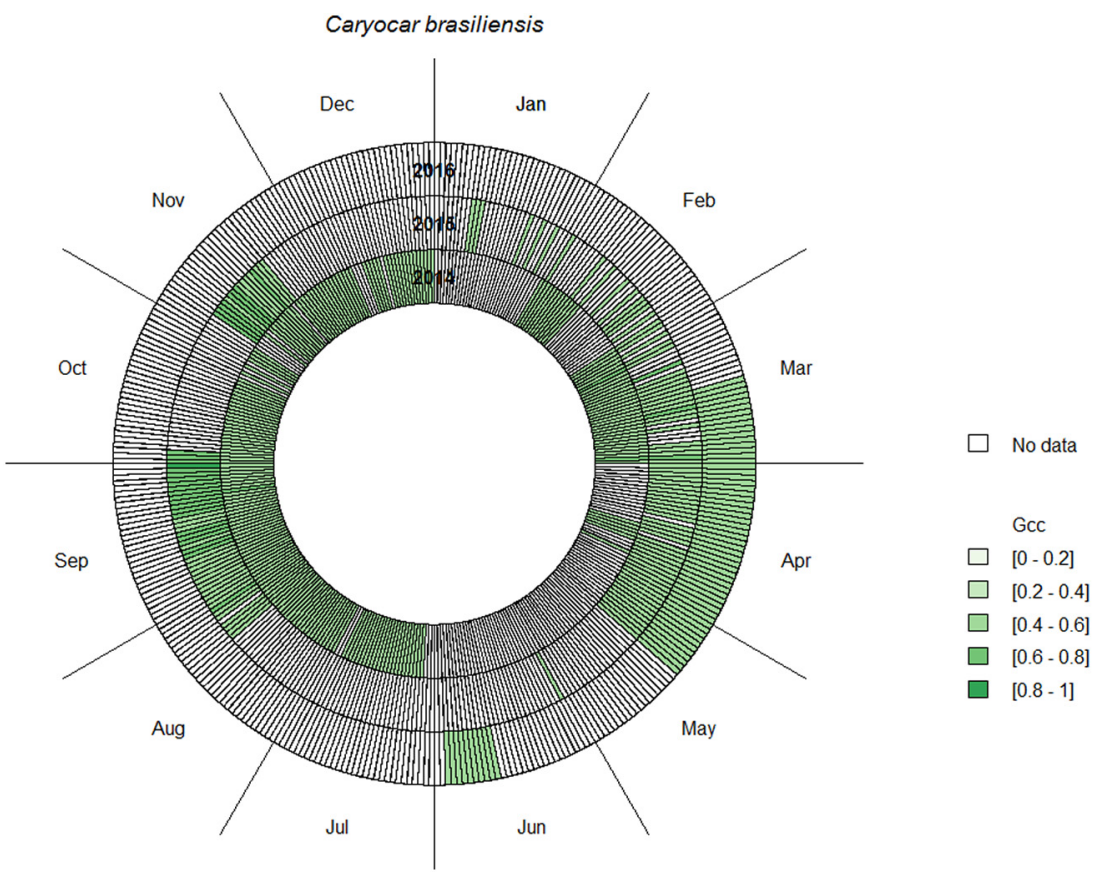

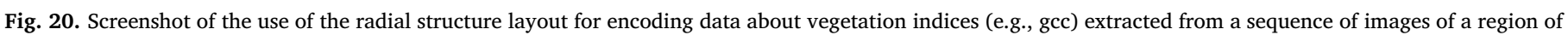
interest (ROI) that represents the species Caryocar brasiliensis (see Fig. 19 for details).

experts. Based on the users' evaluations, we conclude that different strategies for presenting phenological data using both, the radial display and the visual rhythm approach, are promising in the identification of phenological patterns and their correlation with weather data. In the case of weather data, we noticed that we have to offer different options for presentation, depending on the research questions the phenology experts may want to address. Another point is that we have to offer interaction mechanisms to the user choose and change colors to select and represent data of interest.

Similar strategies may be used to present image-related near-remote phenology data collected with cameras of phenocams (Alberton et al., 2017), for example, the variation, over time, of color histograms associated with regions of interest containing particular plant individuals.
Scientific and Technological Development). We thank all members of the Phenology Laboratory at UNESP for their overall support, especially during requirements and prototype development phases. Specially, we would like to thank all members of e-phenology Project for their assistance in the database modeling steps of this work.

\section{References}

Aigner, W., Miksch, S., Schumann, H., Tominski, C., 2011. Visualization of Time-Oriented Data. Human-Computer Interaction Series. Springerhttp://dx.doi.org/10.1007/9780-85729-079-3.

Alberton, B., Almeida, J., Helm, R., Torres, R. da S., Menzel, A., Morellato, L.P.C., 2014. Using phenological cameras to track the green up in a cerrado savanna and its on-theground validation. Eco. Inform. 19 (0), 62-70. http://dx.doi.org/10.1016/j.ecoinf. 
2013.12.011. URL: http://www.sciencedirect.com/science/article/pii/ S1574954113001325.

Alberton, B., Torres, R. da S., Cancian, L.F., Borges, B.D., Almeida, J., Mariano, G.C., dos Santos, J., Morellato, L.P.C., 2017. Introducing digital cameras to monitor plant phenology in the tropics: applications for conservation. Perspec. Ecol. Conserv. 15 (2), 82-90. http://dx.doi.org/10.1016/j.pecon.2017.06.004. URL: http://www. sciencedirect.com/science/article/pii/S2530064417300019.

Almeida, J., Santos, J.A., Alberton, B., Morellato, L.P.C., Torres, R. da S., 2013a. Plant species identification with phenological visual rhythms. In: Proceedings of the 9th IEEE International Conference on eScience (eScience 2013). eScience.

Almeida, J., Santos, J.A., Alberton, B., Morellato, L.P.C., Torres, R. da S., 2013b. Visual rhythm-based time series analysis for phenology studies. In: Proceedings of the 20th IEEE International Conference on Image Processing. ICIP, pp. 1-5.

Almeida, J., dos Santos, J.A., Alberton, B., Morellato, L.P.C., Torres, R. da S., 2016. Phenological visual rhythms: compact representations for fine-grained plant species identification. Pattern Recogn. Lett. 81, 90-100. http://dx.doi.org/10.1016/j.patrec. 2015.11.028. URL: http://www.sciencedirect.com/science/article/pii/ S0167865515004274.

Ankerst, M., Keim, D.A., Peter Kriegel, H-P., November, 1996. Circle segments: a technique for visually exploring large multidimensional data sets. In: Visualization '96, Hot Topic Session, San Francisco, CA, pp. 1996.

Arsenault, R., Ware, C., Plumlee, M., Martin, S., Whitcomb, L., Wiley, D., Gross, T., Bilgili, A., 2004. A system for visualizing time varying oceanographic 3d data. In: Oceans '04. MTTS/IEEE Techno-Ocean '04. Vol. 2. pp. 743-747. http://dx.doi.org/10.1109/ OCEANS. 2004.1405535.

Athayde, E.A., Morellato, L.P.C., 2014. Anthropogenic edges, isolation and the flowering time and fruit set of anadenanthera peregrina, a cerrado savanna tree. Int. J. Biometeorol. 58 (4), 443-454. http://dx.doi.org/10.1007/s00484-013-0727-y.

Burch, M., Diehl, S., 2008. Timeradartrees: visualizing dynamic compound digraphs. Comput. Graphics Forum 27 (3), 823-830. http://dblp.uni-trier.de/db/journals/cgf/ cgf27.html\#BurchD08.

Camargo, M.G.G., Souza, R.M., Reys, P., Morellato, L.P.C., 2011. Effects of environmental condi- tions associated to the cardinal orientation on the reproductive phenology of the cerrado savanna tree Xylopia aromatica (annonaceae). Annal. Braz. Acad. Sci. 83, $1007-1020$

Carlis, J.V., Konstan, J.A., 1998. Interactive visualization of serial periodic data. In: Proceedings of the 11th Annual ACM Symposium on User Interface Software and Technology. ACM; UIST '98, New York, NY, USA, pp. 29-38. http://dx.doi.org/10. $1145 / 288392.288399$.

Chung, M.G., Kim, H., Song, S.M.H., 2000. A scene boundary detection method. In: Proceedings 2000 International Conference on Image Processing (ICIP). vol. 3. pp. 933-936.

Daassi, C., Dumas, M., Fauvet, M.C., Nigay, L., Scholl, P.C., 2013. Visual Exploration of Temporal Object Databases.

d'E ça Neves, F.F., Morellato, L.P.C., 2004. Métodos de amostragem e avaliação utilizados em estudos fenológicos de florestas tropicais. Acta Bot. Bras. 18 (1), 99-108.

Dierenbach, J., Badeck, F.-W., Schaber, J., 2013. The plant phenological online database (PPODB): an online database for long-term phenological data. Int. J. Biometeorol. 57 (5), 805-812. http://dx.doi.org/10.1007/s00484-013-0650-2. (01 Sep, 1432-1254).

dos Santos, L.C.B., Guimarães, S.J.F., Araújo, A.A., dos Santos, J.A., 2014. Unsupervised hyperspectral band selection based on spectral rhythm analysis. In: 27th SIBGRAPI Conference on Graphics, Patterns and Images, SIBGRAPI 2014, Rio de Janeiro, Brazil, August 27-30, 2014, pp. 157-164. http://dx.doi.org/10.1109/SIBGRAPI.2014.51.

Draper, G.M., Livnat, Y., Riesenfeld, R.F., 2009. A survey of radial methods for information visualization. IEEE Trans. Vis. Comput. Graph. 15 (5), 759-776. http://dx. doi.org/10.1109/TVCG.2009.23.

Drocourt, Y., Borgo, R., Scharrer, K., Murray, T., Bevan, S.I., Chen, M., 2011. Tempora visualization of boundary-based geo-information using radial projection. Comput. Graphics Forum. http://dx.doi.org/10.1111/j.1467-8659. 2011.01947.x.

Elmasri, R., Navathe, S., 2010. Fundamentals of Database Systems, 6th ed. AddisonWesley Publishing Company, USA.

Guimarães, S.J.F., Couprie, M., Araújo, A.A., Leite, N.J., 2003. Video segmentation based on 2d image analysis. Pattern Recogn. Lett. 24 (7), 947-957. http://dx.doi.org/10. 1016/S0167-8655(02)00218-0. URL: http://www.sciencedirect.com/science/ article/pii/S0167865502002180.

Ferreira, N., Lins, L.D., Fink, D., Kelling, S., Wood, C., Freire, J., Silva, C.T., 2011. Birdvis: visualizing and understanding bird populations. IEEE Trans. Vis. Comput. Graph. 17 (12), 2374-2383.

Fisher, N.I., Lewis, T., Embleton, B.J.J., 1993. Statistical Analysis of Spherical Data. Cambridge University Press.

Hochman, N., Schwartz, R., 2012. Visualizing Instagram: Tracing Cultural Visual Rhythms.

Horn, M.S., Tobiasz, M., Shen, C., 2009. Visualizing biodiversity with voronoi treemaps. In: Proceedings of the 2009 Sixth International Symposium on Voronoi Diagrams. Washington, DC, USA: IEEE Computer Society; ISVD '09, pp. 265-270. http://dx.doi. org/10.1109/ISVD.2009.22.

Keim, D.A., Schneidewind, J., Sips, M., 2004. Circleview: A New Approach for Visualizing Time-related Multidimensional Data Sets. In: Proceedings of the Working Conference on Advanced Visual Interfaces. ACM; AVI '04, New York, NY, USA, pp. 179-182. http://dx.doi.org/10.1145/989863.989891.

Kim, H., Park, S.J., Lee, J., Kim, W.M., Song, S.M., 1998. Processing of partial video data for detection of wipes. In: Storage and Retrieval for Image and Video Databases VII (1998). Vol. 3656. pp. 280-289. http://dx.doi.org/10.1117/12.333847.

Leite, R.A., Schnorr, L.M., Almeida, J., Alberton, B., Morellato, L.P.C., Torres, R. da S., Comba, J.L., 2016. Phenovis - a tool for visual phenological analysis of digital camera images using chronological percentage maps. Inf. Sci. 372, 181-195. http://www. sciencedirect.com/science/article/pii/S0020025516306235.

Li, J., Meng, Z.P., Huang, M.L., Zhang, K., 2015. An interactive radial visualization of geoscience observation data. In: Proceedings of the 8th International Symposium on Visual Information Communication and Interaction. ACM; VINCI '15, New York, NY, USA, pp. 93-102. http://dx.doi.org/10.1145/2801040.2801061.

Matuszewski, D.J., Lopes, R.M., Cesar, R.M., 2013. Visual rhythm-based method for continuous plankton monitoring. In: IEEE 9th International Conference on e-Science 2013;0, pp. 204-211 doi:10.1109/eScienrce.2013.18.

Mariano, G.C., Staggemeier, V.G., Morellato, L.P.C., Torres, R. da S., Alberton, B., 2016a A visual rhythm approach to visualize multidimensional cyclical data: a case study on phenological data analysis. In: International Conference on Digital Libraries (ICDL).

Mariano, G.C., Morellato, L.P.C., Almeida, J., Alberton, B., de Camargo, M.G.G., Torres, R da S., 2016b. Modeling plant phenology database: blending near-surface remote phenology with on-the-ground observations. Ecol. Eng. 91, 396-408. http://dx.doi. org/10.1016/j.ecoleng.2016.03.001. URL: http://www.sciencedirect.com/science/ article/pii/S0925857416301501.

Mariano, G.C., Soares, N.C., Morellato, L.P.C., Torres, R. da S., 2017. Change frequency heatmaps for temporal multivariate phenological data analysis. In: 2017 IEEE 13th International Conference on e-Science (e-Science), pp. 305-314 doi:10.1109/ eScience.2017.44.

Masoodian, M., Endrass, B., Bühling, R., Ermolin, P., André, E., 2013. Time-pie visualization: Providing contextual information for energy consumption data. In: 2013 17th International Conference on Information Visualisation, pp. 102-107. http://dx.doi. org/10.1109/IV.2013.12.

Matuszewski, D.J., Martins Jr., C.I.O., Strickler Jr., R.M.C., Lopes, R.M., 2012. Visual rhythm-based plankton detection method for ballast water quality assessment. In: 19th IEEE International Conference on Image Processing, ICIP 2012, Lake Buena Vista, Orlando, FL, USA, September 30-October 3, 2012, pp. 1009-1012. http://dx. doi.org/10.1109/ICIP.2012.6467033.

Morellato, L.P.C., Talora, D.C., Takahasi, A., Bencke, C.C., Romera, E.C., Zipparro, V.B., 2000. Phenology of Atlantic rain forest trees: a comparative study. Biotropica 32 (4b), 811-823. http://dx.doi.org/10.1111/j.1744-7429.2000.tb00620.x.

Morellato, L.P.C., Alberti, L.F., Hudson, I.L., 2010a. Applications of circular statistics in plant phenology: a case studies approach. In: Hudson, I.L., Keatley, M.R. (Eds.), Phenological Research. Springer, Netherlands, pp. 339-359 doi:10.1007/978-90481-3335-2 16

Morellato, L.P.C., Camargo, M.G.G., D'Eça Neves, F.F., Luize, B.G., Mantovani, A Hudson, I.L., 2010b. The Influence of Sampling Method, Sample Size, and Frequency of Observations on Plant Phenological Patterns and Interpretation in Tropical Forest Trees. Springer Netherlands, Dordrecht, pp. 99-121. http://dx.doi.org/10.1007/97890-481-3335-2 5

Morellato, L.P.C., Camargo, M.G.G., Gressler, E., 2013. A review of plant phenology in south and central america. In: Schwartz, M.D. (Ed.), Phenology: An Integrative Environmental Science. Springer Netherlands, Dordrecht, pp. 91-113. http://dx.doi. org/10.1007/978-94-007-6925-0_6.

Morellato, L.P.C., Alberton, B., Alvarado, S.T., Borges, B., Buisson, E., Camargo, M.G.G., Cancian, L.F., Carstensen, D.W., Escobar, D.F, Leite, P.T., Mendoza, I., Rocha, N.M., Soares, N.C., Silva, T.S.F., Staggemeier, V.G., Streher, A.S., Vargas, B.C., Peres, C.A., 2016. Linking plant phenology to conservation biology. Biol. Conserv. 195, 60-72. http://dx.doi.org/10.1016/j.biocon.2015.12.033. URL: http://www.sciencedirect. com/science/article/pii/S0006320715302123.

Ngo, C.W., Pong, T.C., Chin, R.T., 1999. Detection of gradual transitions through tem poral slice analysis. In: CVPR. IEEE Computer Society, pp. 1036-1041.

Ngo, C.W., Pong, T.C., Zhang, H., 2003. Motion analysis and segmentation through spatio-temporal slices processing. IEEE Trans. Image Process. 12 (3), 341-355.

Schwartz, M.D., 2003. Phenoclimatic measures phenology: an integrative environmenta science. In: Schwartz, M.D. (Ed.), Phenology: An Integrative Environmental Science. Springer Netherlands, Dordrecht, pp. 331-343. http://dx.doi.org/10.1007/978-94 007-0632-3\21. (volume 39 of Tasks for vegetation science 34 ).

Soares, N.C., Morellato, L.P.C., 2018. Crepuscular pollination and reproductive ecology of trembleya laniflora (melastomataceae), an endemic species in mountain rupestrian grasslands. Flora 238, 138-147. http://dx.doi.org/10.1016/j.flora.2016.12.005. URL: http://www.sciencedirect.com/science/article/pii/S0367253016301980.

Staggemeier, V., Morellato, L.P.C., 2011. Reproductive phenology of coastal plain Atlantic forest vegetation: comparisons from seashore to foothills. Int. J. Biometeorol. 55 (6), 843-854. http://dx.doi.org/10.1007/s00484-011-0482-x.

Talora, D.C., Morellato, L.P.C., 2000. Fenologia de espécies arbóreas em floresta de planície litorânea do sudeste do brasil 1. Rev. Bras. Bot. 3 (1966), 13-26.

Tominski, C., Abello, J., Schumann, H., 2004. Axes-based visualizations with radial layouts. In: Proceedings of the 2004 ACM Symposium on Applied Computing. ACM; SAC '04, New York, NY, USA, pp. 1242-1247. http://dx.doi.org/10.1145/967900. 968153.

Valio, F.B., Pedrini, H., Leite, N.J., 2011. Fast rotation-invariant video caption detection based on visual rhythm. In: Progress in Pattern Recognition, Image Analysis, Computer Vision, and Applications - 16th Iberoamerican Congress, CIARP 2011 , Pucón, Chile, November 15-18, 2011. Proceedings, pp. 157-164. http://dx.doi.org/ 10.1007/978-3-642-25085-9_18.

Vogado, N.O., de Camargo, M.G.G., Locosselli, G.M., Morellato, L.P.C., 2016. Edge effects on the phenology of the guamirim, Myrcia guianensis (myrtaceae), a cerrado tree, Brazil. Trop. Conserv. Sci. 9 (1), 291-312.

Weber, M., Alexa, M., Müller, W., 2001. Visualizing time-series on spirals. In: Proceedings of the IEEE Symposium on Information Visualization. IEEE Computer Society; INFOVIS '01, Washington, DC, USA, pp. 7. URL: http://dl.acm.org/citation.cfm?id= 580582.857719 